\title{
Organische Phosphorverbindungen im Säuglingsharn, ihr Ursprung und ihre Bedeutung für den Stoffwechsel. \\ Von
}

Dr. Arthur Keller, Assistent der Klinik.

(Aus der Universitäts-Kinderklinik zu Breslau.)

(Der Redaction zugegangen am 9. Januar 1899.)

Die Thatsache, dass im Harn ein Theil des Phosphors nicht in Form von Phosphorsäure, sondern in organischer Bindung ausgeschieden wird, ist zwar seit den Arbeiten von Ronalds und von Klüpfel und Fehling bekannt; als derartige Phosphorverbindungen wurden auch bereits von Sotnitschewsky die Glycerinphosphorsäure und von Rockwood die Phosphorfleischsäure nachgewiesen, aber quantitative Bestimmungen der organischen und anorganischen Phosphorverbindungen im Harne liegen aus älterer Zeit nur vereinzelt, so von Lepine und Eymonnet, Zuelzer und Chapelle vor.

In neuerer Zeit wendet sich diesen Fragen erhöhtes Interesse zu, da mannichfache Erfahrungen darauf hindeuten, dass den organischen Phosphorverbindungen eine wichtige Rolle im Stoffwechsel zukommt. Aus Siegfried's Laboratorium stammt eine Arbeit von 0 ertel, ${ }^{1}$ ) der bei einer Anzahl anscheinend normaler erwachsener Menschen die Ausscheidung von Gesammtphosphor und organischem Phosphor im Harn quantitativ bestimmte. Dabei wendet er folgende Methode an: 50 resp. $100 \mathrm{ccm}$. Harn wurden in Silberschalen abgedampft, mit Aetzkali und Salpeter geschmolzen, die salpetersaure Lösung der Schmelze mit molybdänsaurem Ammo-

1) Zeitschrift f. physiol. Chemie, Bd. XXVI, 1898/99, S. 123. 
niak gefällt und die Methode dann in der bekannten Weise weitergeführt bis zum Glühen und Wägen der pyrophosphorsauren Magnesia. In einer zweiten Portion Harn wurden die Phosphate mit Chlorcalium in ammoniakalischer Lösung gefällt, abfiltrirt und gewaschen und im Filtrat der Phosphor wie oben bestimmt, das Ergebniss als organisch gebundener Phosphor angesprochen.

Nach Oertel's Untersuchungen werden $1,6-4,8 \%$ des Gesammtphosphors in organischer Verbindung ausgeschieden, die absoluten Mengen des organischen Phosphors schwanken zwischen 0,12 und $0,03 \mathrm{~g}_{2} \mathrm{O}_{5}$. Das Verhältniss von organischem Phosphor zum Gesammt-Phospor ist nicht nur bei verschiedenen Individuen verschieden, sondern ändert sich auch bei derselben Versuchsperson.

Bei drei Erwachsenen untersuchte Oertel den Einfluss der Arbeit auf die Ausscheidung organischen Phosphors und kam zu dem Resultat, dass ein derartiger Einfluss, wenigstens bei kurzdauernder Arbeit, nicht existirt. Diese Untersuchungen, bei denen er ausser dem Phosphor noch den Stickstoffgehalt des Harns bestimmte, führten ihn aber zu dem weiteren Schluss, dass die Grösse der Ausscheidung des organisch gebundenen Phosphors den Grössen des Stickstoffumsatzes parallel geht.

Wenn auch die Zahlen für das Verhältniss von $\mathrm{N} \mathrm{zu} \mathrm{P}_{2} \mathrm{O}_{5}$ im organisch gebundenen Phosphor thatsächlich nur innerhalb enger Grenzen von 100:0,3 bis 100:0,57 schwanken, so scheint mir doch der Schluss durch Beobachtung von nur drei Individuen nicht genügend gestützt. Auf die Schlussbemerkungen von Oertel komme ich an späterer Stelle zurück, jetzt will ich zunächst über meine eigenen Untersuchungen berichten.

Bei Gelegenheit von Stoffwechseluntersuchungen ${ }^{1)}$ an Säuglingen ergaben sich auffallende Unterschiede in der Phosphorausscheidung beim Brustkind und beim künstlich genährten Kinde, und zwar wird von künstlich genährten Säug-

1) Zeitschr. f. klin. Medicin, Bd. 36, 1898. 
lingen erheblich mehr Phosphorsäure im Harn ausgeschieden, als von Brustkindern. Die Differenz ist so gross, dass sie deutlich genug erkennbar ist, auch wenn erhebliche Unterschiede in Betreff des Ernährungszustandes und des Alters der Kinder, der Menge der aufgenommenen Nahrung und des darin enthaltenen Phosphors bestehen; sie tritt aber um so deutlicher hervor, wenn wir Gelegenheit haben, an demselben Kinde den Einfluss verschiedener Ernährung auf die Ausscheidung von Phosphor zu untersuchen, wie ich dies bei drei Kindern ausgeführt habe.

Diese letzteren Kinder wurden zunächst mit Frauenmilch ernährt und erhielten in einer zweiten, ebenfalls mehrtägigen Periode Kuhmilch mit 2 Theilen Wasser verdünnt. Um vergleichbare Resultate zu haben, wurde den Kindern während beider Perioden täglich die gleiche Menge von Nahrung zugemessen. Ich möchte noch hervorheben, dass wenigstens bei zweien der Versuchskinder zwischen den beiden Perioden nur wenige Tage Zwischenraum waren und dass das Allgemeinbefinden der Kinder, soweit wir klinisch beobachten konnten, sich nicht geändert hatte.

Aus den Untersuchungen ergab sich, dass die Kinder bei Ernährung mit Frauenmilch bedeutend weniger Phosphorsäure im Harn ausschieden als bei Ernährung mit Kuhmilch. Da ich in diesen Fällen ausserdem die Menge der getrunkenen Nahrung kannte und so die Quantität des in der Nahrung eingeführten Phosphors wenigstens annähernd berechnen konnte, stellte sich die weitere Thatsache heraus, dass mit Kuhmilch die Unterschiede im Gehalt des Harns an Phosphorsäure beträchtlich grösser sind, als dem Gehalt der Nahrung an Phosphor entsprechen würde.

Aehnliche Unterschiede, wie sie die absoluten Zahlen der im Harn ausgeschiedenen Phosphorsäuremengen zeigen, treten hervor, wenn wir das Verhältniss $\mathrm{P}_{2} \mathrm{O}_{5}$ zu $\mathrm{N}$ im Harn bei Brustkindern und bei künstlich genährten Kindern berücksichtigen. Bei den ersteren war dasselbe im Durchschnitt 1:7, bei den letzteren etwa 1:2.

Wenn wir nun erwägen, dass das Verhältniss von $\mathrm{P}_{2} \mathrm{O}_{5}$ 
zu $\mathrm{N}$ in der Frauenmilch ungefähr 1:5,5, in der Kuhmilch $1: 2,5$ ist, so erscheint der Schluss gerechtfertigt, dass die Ausscheidung der Phosphorsäure nicht allein von der Quantität des in der Nahrung eingeführten Phosphors abhängig ist, sondern dass dabei noch andere Momente in Frage kommen. Da nun Frauenmilch und Kuhmilch sich nicht nur im Gehalt an Gesammtphosphor, sondern namentlich an organischem Phosphor unterscheiden, so lag der Gedanke nahe, dass die Art der Bindung des Phosphors in der Nahrung auf die Ausnutzung und Ausscheidung desselben Einfluss hat.

Um der Entscheidung der berührten Fragen näher zu kommen, habe ich an gesunden und kranken Kindern des ersten Lebensjahres bei verschiedenartiger Ernährung Stoffwechseluntersuchungen in der Weise ausgeführt, dass in der Nahrung, im Harn und Koth Stickstoff und Phosphor bestimmt wurde.

Beim Phosphorstoffwechsel musste für mich die Bestimmung, in welcher Form der Phosphor im Harn ausgeschieden wird, von demselben Interesse sein, wie bei den Untersuchungen über Ausnutzung des Nahrungsstickstoffs nicht nur die Ausscheidung von Gesammtstickstoff im Harn, sondern auch die von Harnstickstoff Berücksichtigung findet.

Aus diesem Grunde habe ich neben dem Gesammtphosphor gleichzeitig die Menge des im Harn ausgeschiedenen organischen Phosphors bestimmt.

Da unsere Kenntnisse betreffs der Ausscheidung organischer Phosphorverbindungen im Harn, wie aus der oben angeführten Litteratur hervorgeht, sehr mangelhaft sind, dürften meine Untersuchungen gleichzeitig für das Studium dieser speciellen Frage von Bedeutung sein.

Denn dadurch; dass ich nicht nur organischen und Gesammtphosphor im Harn, sondern gleichzeitig Stickstoff und Phosphor in Nahrung und Koth bestimmte, hatte ich eine Reihe von Zahlen in der Hand, die für die Beurtheilung des Einflusses bestimmter Stoffwechselvorgänge auf die Ausscheidung organischen Phosphors im Harn von Wichtigkeit sind. Wenn in dieser Hinsicht schon die Ergebnisse meiner Untersuchungen 
auch für die allgemeine Physiologie, nicht nur für die der Kinder Werth haben, so kommt noch dazu, dass Versuche an Kindern für die Entscheidung der in Rede stehenden Frage besonders geeignet sind. In den gebräuchlichsten Nahrungsmitteln für Säuglinge, in der Frauenmilch und in der Kuhmilch, haben wir erhebliche Gegensätze in Betreff des Phosphorgehalts. Die Kuhmilch enthält ungefähr 2,4 g $\mathrm{P}_{2} \mathrm{O}_{5}$ im Liter, davon sind $0,67 \mathrm{~g}$ organisch gebunden, während die Frauenmilch nur $0,47 \mathrm{~g} \mathrm{P}_{2} \mathrm{O}_{5}$ im Liter enthält, von denen nur ein minimaler Theil aus organischen Salzen besteht. Ausser Frauenmilch und Kuhmilch in verschiedenen Verdünnungen habe ich noch bei Ernährung mit Malzsuppe und bei Zusatz von Natriumphosphat zur Frauenmilch Untersuchungen angestellt. Auch in anderer Beziehung waren die Versuchsanordnungen möglichst verschiedenartig: die Kinder gehörten verschiedenen Altersstufen des ersten Lebensjahres an, zwei davon waren gesunde, normal gedeihende Brustkinder, die andern litten an mehr oder weniger schweren Ernährungsstörungen, sodass namentlich unter dem Einfluss verschiedenartiger Ernährung auch grosse Differenzen in Betreff von Körpergewichtszunahme von $\mathrm{N}$ - und $\mathrm{P}_{2} \mathrm{O}_{5}$-Ansatz und in Betreff der Menge der in Harn ausgeschiedenen Gesammtphosphorsäure zwischen den einzelnen Versuchen bestehen. Schliesslich möchte ich noch hervorheben, dass in mehreren Fällen Versuche an einem Kinde bei verschiedenartiger Ernährung ausgeführt wurden, Versuche, aus deren Ergebniss wir wohl am ehesten den Einfluss von Menge und Art der Nahrung auf die Ausscheidung organischen Phosphors ermessen können.

Betreffs der Versuchsanordnung, des Auffangens von Harn und Koth verweise ich auf die Angaben in meinen früheren Arbeiten ${ }^{1}$ ) und auf die Arbeit von Freund. ${ }^{2}$ ) Ich möchte auch hier erwähnen, dass es bei unserer Methodik mit Sicherheit gelingt, die Excrete ohne Verlust aufzufangen. Was die Bestimmung des Gesammtphosphors in Nahrung und Koth

1) Centralblatt für innere Medicin, 1898, Nr. 21 und Jahrbuch für Kinderheilkunde, 44. Band, 1897, S. 25.

2) Jahrbuch für Kinderheilkunde, 48. Band, 1898. 
betrifft, habe ich die Methode von Neumann gewählt und zwar mit geringen Abweichungen, wie sie im Laboratorium von Herrn Professor Röhmann üblich sind, dem ich für seine liebenswürdigen Rathschläge betreffs der Untersuchungsmethodik auch an dieser Stelle besten Dank sage. Die Methode ist in den Arbeiten von Marcuse ${ }^{1}$ ) und von Steinitz²) angegeben. Da ich in der Ausführung auf Rath von Herrn Professor Röhmann einige Aenderungen, die sich im Laboratorium bereits bewährt hatten, anwendete, will ich die Methode hier ausführlich angeben.

Eine gewogene Menge von Substanz, sei es Koth oder Milch oder auch Harn, wird im Kjeldahl-Kolben mit etwa $10 \mathrm{ccm}$. rauchender Salpetersäure langsam erhitzt, bis die braunen Nitrosodämpfe verschwunden sind, nach dem Abkühlen wurden von concentrirter Schwefelsäure etwa $10 \mathrm{ccm}$. zugesetzt und die Flüssigkeit wiederum erhitzt. Nach jedesmaligem Abkühlen wurden 2-3 Portionen von Ammonnitrat (an Menge im Ganzen etwa soviel Gramm, wie Cubikcentimeter Schwefelsäure verwendet wurde) zugesetzt und die Flüssigkeit allmählich immer stärker erhitzt, zuletzt mit einem Dreibrenner. Stärkeres andauerndes Erhitzen habe ich, wenn es sich um grössere Mengen von Substanz handelte, vermieden, bevor nicht Ammonnitrat zugesetzt war, da vorher beim Erhitzen leicht starkes Stossen eintritt. Die nach dem Zusatz der Schwefelsäure unter dem Einfluss des Erwärmens schwarz gefärbte Flüssigkeit wird allmählich immer heller, die Verbrennung ist genügend weit durchgeführt, wenn die Flüssigkeit farblos ist. Man thut übrigens gut, nicht allzuviel Ammonnitrat (die nothwendige Menge kann man in jedem einzelnen Falle unter Beobachtung der' allmählich sich vollziehenden Farbenveränderung abschätzen) zu verwenden oder die Flüssigkeit zu stark einzudampfen, da sonst beim Erkalten leicht das Salz ausgeschieden wird, welches oft dem Kjeldahl-Kolben fest anhaftet. Aus dem Kolben wird die Flüssigkeit nach dem Erkalten mit destillirtem Wasser sorgfältig in ein Becherglas

1) Pflüger's Archiv f. die ges. Physiologie, Bd. 67, 1897, S. 363.

2) Pflüger's Archiv f. die ges. Physiologie, Bd. 72. 
herausgespült, Ammoniak bis zu alkalischer Reaction und dann Salpetersäure bis zu stark saurer Reaction zugefügt und unter starkem Umrühren die nöthige Menge von Molybdänlösung zugesetzt. Bei der weiteren Ausführung der Bestimmungen bis zum Glühen des Magnesium-Pyrophosphates bin ich im Grossen und Ganzen Fresenius ${ }^{1}$ ) gefolgt.

Die Bestimmung des Gesammtphosphors im Harn wurde in derselben Weise ausgeführt, wie für Milch und Koth oben angegeben. Je nach der Menge des Phosphorgehaltes wurden 50-200 ccm. Harn zur Bestimmung verwendet und gleich im Anfang nach dem Zusatz von Salpetersäure und vor dem Zusatz von Schwefelsäure auf ein geringes Volumen (etwa $15 \mathrm{ccm}$.) eingedampft.

Auch die anorganischen Phosphate wurden durch die Wägemethode bestimmt. Ich wollte es vermeiden, für die Bestimmung des Gesammtphosphors eine Wägemethode und für die der Phosphate eine Titrirmethode anzuwenden, da gerade für die Bestimmung von organischem Phosphor, der nur in sehr geringer Menge vorhanden ist und ausserdem nur aus der Differenz von Gesarnmtphosphor und anorganischen Phosphaten berechnet wird, die Anwendung einer und zwar exacten Methode nothwendig ist.

Die Harnmenge beim Säugling ist zu gering, als dass eine einzelne Tagesmenge für je 2 Parallelbestimmungen von anorganischem und Gesammtphosphor ausreichen würde, namentlich wenn man beim geringen Phosphorgehalt des Harns von Brustkindern gezwungen ist, 100-200 ccm. für jede Bestimmung zu verwenden. Ich habe in Folge dessen die Harnmengen der einzelnen Tage während der Dauer eines Versuches vereinigt und diesen Mischharn zu den Phosphorbestimmungen verwendet. Da sämmtliche Versuche mit Ausnahme eines einzigen 5 Tage dauerten, stellen meine Zahlen den Durchschnitt einer 5tägigen Periode dar. Dieser Umstand, dass wir es nicht mit Zahlen, die aus der Untersuchung einer einzelnen Tagesmenge sich ergeben, zu thun haben, erhöht meines Erachtens nur den Werth meiner Untersuchungen.

1) Anleitung zur quantitativen Analyse, II. Band, S. 691. 
In der Tabelle*) I gebe ich eine Uebersicht über die Resultate meiner Untersuchungen. Während ich die Krankengeschichten der zu den Versuchen verwendeten Kinder, sowie die Belege für die aus den Phosphorbestimmungen im Harn sich ergebenden Zahlen am Schluss der Arbeit mittheilen und eine ausführliche Besprechung der Stoffwechselversuche, soweit es . sich um die Resorption und Retention von $\mathrm{N}$ und $\mathrm{P}_{2} \mathrm{O}_{5}$ handelt, an anderer Stelle beabsichtige, gebe ich in meiner Tabelle das Alter der Kinder an, das Körpergewicht bei Beginn des Versuches, die durchschnittliche tägliche Körpergewichtszunahme und Retention von $\mathrm{P}_{2} \mathrm{O}_{5}$. In der zweiten Columne bezeichne ich die Versuchskinder mit Zahlen, um auszudrücken, welche Versuche an demselben Kind ausgeführt sind. Sämmtliche Zahlen, die die Untersuchung des Harns und der Nahrung betreffen sind Mittelzahlen, wie sie aus den Ergebnissen des ganzen Versuches für den einzelnen Tag berechnet sind. Ich habe zur Erklärung der Tabelle wohl nichts weiter hinzuzufügen als das eine, dass sämmtliche Kinder in 24 Stunden 5 Mahlzeiten erhielten, nur in Versuch I und IX wurden zum Zweck anderweitiger Untersuchungen 10 Mahlzeiten pro Tag verabreicht.

Bei der Anordnung der Versuche wurde übrigens nicht darauf allein Rücksicht genommen, wie man am besten äie Bedingungen studiren kann, von denen die Ausscheidung organisch gebundenen Phosphors im Harn abhängig ist, sondern ich ging bei den Versuchen darauf aus, die verschiedenen Vorgänge im Phosphorstoffwechsel des Säuglings überhaupt zu erforschen.

Aus den Zahlen der Tabelle geht hervor, dass die absolute Menge des im Harn ausgeschiedenen organischen Phosphors bei verschiedenen Kindern ziemlich erhebliche Schwankungen zeigt von $0,00218-0,0167 \mathrm{~g} \mathrm{P}_{2} \mathrm{O}_{5}$. Bei den drei gesunden Kindern fand ich $0,0081-0,0061$ und $0,00596 \mathrm{~g} \mathrm{P}_{2} \mathrm{O}_{5}$ in organischer Bindung enthalten. Wir sehen also, dass die absoluten Zahlen erheblich geringer sind, als diejenigen, welche Oertel für den Erwachsenen angibt (ungefähr $0,05 \mathrm{~g} \mathrm{P}_{2} \mathrm{O}_{5}$, im Maximum 0,12, im Minimum 0,03 g). Wenn wir die Körpergewichte der

*) Die Tabellen sind im Anhang mitgetheilt.

Hoppe-Seyler's Zeitschrift f. physiol. Chemie. XXIX. 
Erwachsenen und der Säuglinge berücksichtigen, so ergibt sich, dass im Verhältniss zum Körpergewicht vom gesunden Kinde erheblich mehr organischer Phosphor im Harn ausgeschieden wird, als vom gesunden Erwachsenen. Dies würde mit der Beobachtung Oertel's übereinstimmen, dass die Grösse der Ausscheidung des organisch gebundenen Phosphors mit der Grösse des Stickstoffumsatzes einhergeht, da wir aus einer genügend grossen Zahl von Stoffwechseluntersuchungen wissen, dass beim wachsenden Organismus die Grösse des Stickstoffumsatzes im Verhältniss zum Körpergewicht bedeutender ist, als beim Erwachsenen. Bei den mannigfachen Verschiedenheiten, die zwischen dem Stoffwechsel des Kindes und des Erwachsenen nicht nur im Betreff der Grösse, sondern auch der Art des Stoffwechsels bestehen, erscheint es mir nicht gerechtfertigt, diese eine Zahl, nämlich die Grösse des Stickstoffumsatzes, zu der Ausscheidung organischen Phosphors in Beziehung zu setzen.

Wenn wir die Grösse der Phosphorausscheidung beim Erwachsenen und beim Kind untereinander vergleichen wollen, erscheinen mir besonders jene Zahlen werthvoll, welche uns angeben, wieviel $\mathrm{P}_{2} \mathrm{O}_{5}$ im organischen Phosphor auf 100 Theile Gesammtphosphor kommen resp. auf 100 Theile Stickstoff. Wir sehen aus den Zahlen der Tabelle, dass gerade in Betreff dieser Zahlen zwischen einzelnen Kindern erhebliche Differenzen bestehen, und zwar finden wir 0,51-9,9\% des Gesammtphosphors in organischer Bindung enthalten. Nach den Untersuchungen von Oertel liegen beim Erwachsenen die entsprechenden Zahlen zwischen 1,6 und 4,8\%.

Bei der weiteren Besprechung will ich vom Vergleich meiner Zahlen mit den beim Erwachsenen gefundenen absehen und nur auf Grund der Ergebnisse meiner Untersuchungen der Frage näher treten, von welchen Bedingungen die Grösse der Ausscheidung organischen Phosphors im Harn beim Kinde abhängig ist. Bei der Untersuchung an Säuglingen haben wir den Vortheil, dass wir jene Momente, denen beim Erwachsenen von jeher ein besonderer Einfluss auf den Phosphorwechsel zugeschrieben worden ist, wie die Menge der während der 
Untersuchungszeit geleisteten körperlichen oder geistigen Arbeit u. s. w., bei unsern Säuglingen vernachlässigen können, da in dieser Richtung zwischen den einzelnen Individuen kaum erhebliche Differenzen bestehen werden. Um so besser werden wir beurtheilen können, in wie weit die Ausscheidung des organischen Phosphors von der Grösse und Art des Stoffwechsels, von der Menge des in der Nahrung eingeführten Phosphors und der Natur der in der Nahrung enthaltenen Phosphorverbindungen und schliesslich von dem Gesundheitszustand der Kinder abhängig ist.

Um diese Frage gesondert besprechen zu können, habe ich meine Untersuchungsergebnisse in einer Reihe besonderer Tabellen zusammengestellt, in denen nach Möglichkeit nur das eine oder andere Moment Berücksichtigung findet, da bei der grossen Menge von Zahlen die Tabelle I wenig übersichtlich erscheint. Ich habe in Tabelle II meine Zahlen nach der Menge des in der Nahrung eingeführten Phosphors geordnet. Im Grossen und Ganzen ist dies gleichzeitig, wie wir uns überzeugen können, ein Maassstab für die Grösse des Stoffwechsels überhaupt, wenn wir den einen Versuch VII unberücksichtigt lassen, bei dem der Nahrung (Frauenmilch) Natriumphosphat zugesetzt wurde und so künstlich ganz besondere Verhältnisse des Phosphorstoffwechsels geschaffen wurden.

Aus den Zahlen dieser Tabelle scheint mir mit genügender Sicherheit hervorzugehen, dass die Grösse des Stickstoffumsatzes, soweit wir sie aus dem Stickstoffgehalt von Nahrung und Harn erschliessen können, keinen erheblichen Einfluss auf die Grösse der Ausscheidung organischen Phosphors im .Harn ausübt. Denn die Zahlen für die absolute Menge des organischen Phosphors und die für das Verhältniss von organischem Phosphor zum Gesammtphosphor gehen weder den Stickstoffzahlen noch den für Phosphor in Harn und Nahrung gefundenen Zahlen parallel.

Zur 'weiteren Charakteristik des Stoffwechsels habe ich in Tabelle III die Versuche nach den aus der Untersuchung der Retention von Phosphor im Organismus sich ergebenden 
Zahlen geordnet und in Tabelle IV nach der Grösse der Körpergewichtszunahme.

Ein Blick auf die betreffenden Zahlenreihen zeigt uns, dass von einem Parallelgehen der absoluten resp. Verhältnisszahlen für die Ausscheidung organischen Phosphors mit denen der täglichen $\mathrm{P}_{2} \mathrm{O}_{5}$-Retention oder mit denen der Körpergewichtszunahme keine Rede ist. Wir mussten also schliessen, dass die Grösse des Umsatzes von Stickstoff und Phosphor im Körper auf die Ausscheidung von organischem Phosphor keinen Einfluss hat.

Um aus der Menge der zur Verfügung stehenden Zahlen doch noch ein klareres Bild zu bekommen, welche Momente auf die Grösse der Ausscheidung von organischen Phosphorverbindungen im Harn einen Einfluss haben, habe ich schliesslich noch zwei Tabellen zusammengestellt, in deren ersterer (Tab. V) die Versuche nach der Höhe der absoluten Zahlen der Ausscheidung organischen Phosphors geordnet sind, je nach dem mehr oder weniger organischer Phosphor im Verhältniss zum Gesammtphosphor im Harn enthalten ist.

Die Zahlen dieser Tabelle zeigen dass die Grösse der Ausscheidung organischen Phosphors bis zu einem gewissen Grade von der Art der Nahrung abhängig ist, insofern als im Allgemeinen bei Ernährung mit Kuhmilch höhere Zahlen sich finden als bei Ernährung mit Frauenmilch. Wir sehen allerdings, dass in der Kuhmilch, auch in der verdünnten, stets mehr, zum Theil erheblich mehr Phosphor in der Nahrung eingeführt wird, als in der Frauenmilch. Aus dieser Reihe fallen zwei Versuche auf: der Versuch. XII und X. In dem ersten wird auch bei Ernährung mit Kuhmilch nicht mehr organischer Phosphor ausgeschieden als von den Brustkindern; es handelt sich jedoch um ein normal gedeihendes Kind. Und im zweiten scheidet das Kind bei Ernährung mit Malzsuppe trotz ziemlich grosser Mengen von Nahrungs- $\mathrm{P}_{2} \mathrm{O}_{5}$ weniger organischen Phosphor aus, als selbst die Brustkinder.

Schliesslich will ich noch erwähnen, dass die absolute Menge des organischen Phosphors im Harn durch den 
Zusatz von Natriumphosphat zur Nahrung nicht beeinflusst wird.

Dass die Art der Nahrung thatsächlich von wesentlicher Bedeutung ist, sehen wir am besten, wenn wir Tabelle VI betrachten: Bei Ernährung mit Frauenmilch wird im Allgemeinen mehr organischer Phosphor im Verhältniss zum Gesammtphosphor ausgeschieden, als bei Ernährung mit Kuhmilch. Wir könnten leicht daran denken, dass dies seinen Grund darin hat, weil sich in der Frauenmilch fast sämmtlicher Phosphor in Form von organischen Verbindungen befindet, während die Kuhmilch erhebliche Mengen von Phosphaten enthält. Es widerspricht dieser Erklärung nur der eine Fall X, in dem bei Ernährung mit Malzsuppe, die doch mehr organische Phosphorverbindungen enthält als eine in demselben Verhältniss verdünnte Kuhmilch, nur $0,51 \%$ des Gesammtphosphors in organischer Bindung ausgeschieden wird.

Inwieweit die Ausscheidung organischen Phosphors im Harn von der Grösse des allgemeinen Stoffwechsels, von der Art der Ernährung u. s. w. abhängig ist, diese Fragen würden leichter zu beantworten gewesen sein, wenn mir eine Reihe von physiologischen Zahlen, gewonnen aus der Untersuchung gesunder Kinder, zur Verfügung gestanden hätte. Leider war mir dazu nur in den 3 Fällen Gelegenheit geboten und ich war darum gezwungen, in meine T'abellen auch die an kranken Kindern gewonnenen Zahlen einzustellen. Um aber die letzteren verwerthen zu können, wäre der Nachweis erforderlich, welchen Einfluss der Gesundheitszustand des Individuums auf die Ausscheidung organischer Phosphorverbindungen hat. Bei Betrachtung der verschiedenen Tabellen, namentlich von Tabelle $\mathrm{V}$ und VI, tritt die bemerkenswerthe Thatsache hervor, dass das kranke Kind weniger organische Phosphorverbindungen im Harn ausscheidet als das gesunde bei der gleichen Ernährung. Diese Beobachtung erklärt uns zum Theil wenigstens die Widersprüche, auf die ich bei der Besprechung der einzelnen Tabellen immer wieder stiess und die auch durch die Verschiedenartigkeit des Untersuchungsmaterials nicht genügend begründet erschienen. Wenn die gesunden Säuglinge relativ mehr orga- 
nischen Phosphor im Harn ausscheiden als die magendarmkranken Kinder bei derselben Ernährung, so können die organischen Phosphorverbindungen nicht aus der eingeführten Nahrung allein stammen, zumal wir aus den Untersuchungen der Ammoniakausscheidung wissen, dass bei den Ernährungsstörungen der Säuglinge eine Verminderung der Oxydationsfähigkeit des Organismus besteht. Wir werden also zu dem Schluss gedrängt, dass die im Harn erscheinenden organischen Phosphorverbindungen auch einer anderen Quelle als der Nahrung entstammen und zum Theil aus dem Zerfall von Körpersubstanz herrühren oder im Körper entstehen.

Mit dieser Annahme würde sich das Verhalten der Ausscheidung organischen Phosphors im Harn bei verschiedener Ernährung vollständig vereinbaren. Die Höhe der Procentzahlen, die das Verhältniss von organischem Phosphor zum Gesammtphosphor im Harn bezeichnen, ist bei den Brustkindern in Wesentlichen dadurch bedingt, dass die Menge des Gesammtphosphors bei Ernährung mit Frauenmilch im Vergleich zu der mit Kuhmilch stark absinkt, während die Ausscheidung organischen Phosphors nicht in demselben Maasse vermindert wird. Wenn nun der Theil der organischen Phosphorverbindungen, der nicht aus der Nahrung stammt, bei verschiedenen Kindern ungefähr gleich ist, so würde sich erklären, dass die Ausscheidung des organischen Phosphors nicht in derselben Weise wie die des Gesammtphosphors steigt und sinkt. Denn der Einfluss der Ernährungsart kann unter diesen Umständen nicht so deutlich hervortreten, als wenn der im Harn erscheinende organische Phosphor vollständig aus nicht oxydirten organischen Verbindungen der Nahrung herrührt.

Die Frage, ob die organischen Phosphorverbindungen des Harns aus der Nahrung allein kommen, lässt sich direkt durch Versuche, bei denen keine Nahrung oder phosphorfreie Nahrung dem Körper zugeführt wird, beantworten. Ueber diese Versuche will ich im Folgenden berichten.

Bei einem 11 Monate alten Kinde, das wegen einer Magendarmerkrankung in die Klinik aufgenommen wurde und bei dem aus therapeutischen Gründen eine absolute Wasserdiät 
zur Leerstellung des Darmes indicirt war, habe ich während dieser Zeit den Harn untersucht, und zwar wurde am 24. Juli, Nachmittags 3 Uhr, der Harnrecipient angelegt, nachdem das Kind seit dem 23. Juli früh, also seit ungefähr 30 Stunden, keine Nahrung, ausser Wasser mit Saccharin versüsst, erhalten hatte. Dieselbe Diät wurde bis zum 25. Juli, Nachmittags 3 Uhr, beibehalten und ebenso lange Zeit blieb der Recipient liegen. Es wurden $620 \mathrm{ccm}$. Harn ausgeschieden, darin waren enthalten:

Als Gesammtphosphor $\quad 0,4308 \mathrm{~g}$

in Form von Phosphaten 0,4247 ,

also in organischer Form $0,0061 \mathrm{~g}=1,4 \%$ des Gesammtphosphors.

Nach den Erfahrungen über die Schnelligkeit, mit der die Nahrung beim Säugling den Darm passirt, sowie aus der Beobachtung der Veränderungen im Aussehen des Stuhles bei unserem Kinde durfte ich annehmen, dass zu der Zeit, als der Harnrecipient angelegt wurde, die letzten Reste von Nahrung aus dem Darm verschwunden waren, und dass der aufgesammelte Harn in Wirklichkeit dem Hungerzustande entspricht.

Um mich nicht wiederholen zu müssen, will ich über die weitere Durchführung des Versuches in chronologischer Reihenfolge berichten. An den beschriebenen Versuch wurde nämlich ein zweiter angeschlossen, um zu constatiren, ein wie grosser Theil des Kothphosphors aus der Nahrung und wie viel aus dem Zerfall von Darmepithelien und aus den Verdauungssäften stammt. Da nun eine Wasserdiät in diesem Falle keine günstigen Bedingungen für die Entscheidung der Frage bot, weil die Ausscheidung der Verdauungssäfte in hohem Grade von der Zuführung der Nahrung abhängig ist, gab ich dem Kinde eine Nahrung, welche keinen Phosphor oder wenigstens nur minimale Mengen enthält, welche aber, wie aus den Körpergewichtswägungen hervorging, ungefähr ausreichend war, um das Kind im Körpergleichgewicht $\mathrm{zu}$ erhalten. Ich verwendete Protogen, eine Eiweissart, welche nach den Angaben von 
Blum ${ }^{1}$ ) aus dem Eiweiss von frischen Hühnereiern durch Behandlung mit Formaldehyd hergestellt wird.

$0,0125 \mathrm{~g}$
0,0128 ) im Mittel $0,01265 \mathrm{~g}$ Magnesiumpyrophosphat $=0,00809 \mathrm{~g} \mathrm{P}_{2} \mathrm{O}_{5}$.

Nach der $2^{1 / 2}$ tägigen Periode absoluter Wasserdiät wurde der Versuch in der Weise weitergeführt, dass das Kind am 25. Juli im Laufe des Nachmittags und der Nacht eine Lösung von 5,5 g Protogen als Nahrung erhielt, am 26. und 27. eine Lösung von je $10 \mathrm{~g}$ Protogen. Die mit Saccharin versüsste Nahrung wurde gut getrunken.

Am 27. Juli, Abends 7 Uhr, erhielt das Kind zum letzten Mal Protogen, von da an wieder Saccharinwasser. Der Harnrecipient blieb bis zum 28. Juli, Nachmittags $3 \mathrm{Uhr}$, liegen; es war also anzunehmen, dass der der Protogendarreichung entsprechende Harn bis zu dieser Zeit vollständig entleert war. In den drei Tagen hatte das Kind im Ganzen 0,020227 $\mathrm{g}$ $\mathrm{P}_{2} \mathrm{O}_{5}$ erhalten, also pro Tag im Durchschnitt 0,00674 g $\mathrm{P}_{2} \mathrm{O}_{5}$.

Während der Protogenperiode wurden entleert:

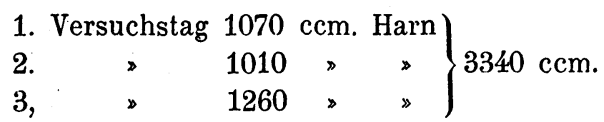

Um für jede einzelne Bestimmung des Phosphors möglichst grosse Quantitäten von Harn verwenden zu können, wurden auch in diesem Versuch die Tagesportionen zu einem Mischharn vereinigt, in denen die Bestimmungen ausgeführt wurden. Nach den Resultaten derselben ergeben sich pro Tag $1113 \mathrm{ccm}$. Harnmenge, darin sind enthalten:

$1,6756 \mathrm{~g}$ Stickstoff, $0,2861 \mathrm{~g} \mathrm{P}_{2} \mathrm{O}_{5}$ als Gesammtphosphor, $0,0084 \mathrm{~g} \mathrm{P}_{2} \mathrm{O}_{5}$ als organischer Phosphor $=2,9 \%$ des Gesammtphosphors.

Im Uebrigen ist hervorzuheben, dass ich bei der Untersuchung dieses Harns, sowohl für die Bestimmung des Gesammtphosphors wie für die der anorganischen Phosphate, je 4 Kontrollbestimmungen und noch dazu in 200 bis $500 \mathrm{ccm}$. Harn ausführte, um meiner Zahlen sicher zu sein. Die Parallelbestimmungen ergaben gut übereinstimmende Werthe.

1) Zeitschr. f. phys. Chemie, Bd. XXII, 1896/97, S. 126. Protogen wird von den Farbwerken, vormals Meister, Lucius u. Brüning hergestellt. 
Nach der Protogenperiode blieb der Harnrecipient noch 24 Stunden länger, also bis zum 29. Juli, Nachmittags, liegen. Da 20 Stunden seit der letzten Mahlzeit bis zum Beginn des Harnaufsammelns vergangen waren, ist anzunehmen, dass der in den 24 Stunden vom 28. zum 29. Juli entleerte Harn dem Hungerzustand entspricht.

Die Untersuchung des Harns ergab in $1520 \mathrm{ccm}$. Tagesmenge :

$1,4098 \mathrm{~g} \mathrm{~N}$ und $0,2140 \mathrm{~g} \mathrm{P}_{2} \mathrm{O}_{5}$ als Gesammtphosphor. 0,0081 g $_{2} \mathrm{O}_{5}$ als organischer Phosphor $=3,8 \%$ des Gesammtphosphors.

Als bei demselben Kind nach einiger Zeit das Allgemeinbefinden und die Magendarmerscheinungen wiederum eine Entleerung des Darmes erforderlich machten, habe ich auch diese 2. Hungerperiode zur Untersuchung von Harn und Koth benutzt. Am 10. August, Abends 8 Uhr, erhielt das Kind die letzte Mahlzeit Malzsuppe, von da an wiederum nur Saccharinwasser. Am 12. August, früh $8 \mathrm{Uhr}$, also nach 36 stündiger Wasserdiät, wurde der Recipient angelegt. Um zu beobachten, in welcher Weise die Zusammensetzung des Harns sich im Verlauf der Hungerperiode ändert, wurde der Harn der ersten 12 Stunden bis zum 12. August, Abends $8 \mathrm{Uhr}$, gesondert aufgefangen und dann weiter 24 Stunden bis zum 13. August. In den ersten 12 Stunden wurden $700 \mathrm{ccm}$. Harn ausgeschieden, deren Untersuchung ergab:

$0,1849 \mathrm{~g}_{8} \mathrm{O}_{5}$ als Gesammtphosphor, $0,0049 \mathrm{~g} \mathrm{P}_{2} \mathrm{O}_{5}$ als organischer Phosphor $=2,3 \%$ des Gesammtphosphors.

Wïhrend des letzten Tages wurde absichtlich dem Kind weniger Saccharinwasser angeboten, um einen concentrirten Harn zu erhalten und so das umständliche Einengen grösserer Harnmengen, wie es für die Bestimmung der Phosphorsäure in stark verdünntem Harn nothwendig ist, zu vermeiden. · In den 24 Stunden wurden in $820 \mathrm{ccm}$. Harnmenge entleert:

$1,3776 \mathrm{~g} \mathrm{~N}$ und $0,3293 \mathrm{~g} \mathrm{P}_{2} \mathrm{O}_{5}$ als Gesammtphosphor, $0,0074 \mathrm{~g} \mathrm{P}_{2} \mathrm{O}_{5}$ als organischer Phosphor $=2,2 \%$ des Gesammtphosphors.

Der Uebersichtlichkeit wegen stelle ich die aus diesem Versuch sich ergebenden Zahlen in einer Tabelle zusammen. 


\begin{tabular}{|c|c|c|c|c|c|c|}
\hline \multirow[b]{2}{*}{ Datum } & \multirow{2}{*}{$\begin{array}{l}\text { Harnmenge } \\
\text { in } \\
24 \text { Stunden }\end{array}$} & \multirow{2}{*}{$\begin{array}{c}\text { Menge } \\
\text { des } \\
\text { Stickstoffs }\end{array}$} & \multirow{2}{*}{$\begin{array}{c}\text { Menge } \\
\text { des } \\
\text { Gesammt- } \\
\text { phosphors }\end{array}$} & \multicolumn{2}{|c|}{ Organischer Phosphor } & \multirow[b]{2}{*}{ Bemerkungen } \\
\hline & & & & $\begin{array}{c}\text { absolute } \\
\text { Menge }\end{array}$ & $\mid \begin{array}{c}\% \text { des } \\
\text { Gesammt } \\
\mathrm{P}_{2} \mathrm{O}_{5}\end{array}$ & \\
\hline 24.-25. Joli & $620 \mathrm{ccm}$. & 1,725 & $0,2081 \mathrm{~g}$ & $0,0061 \mathrm{~g}$ & $1,4 \%$ & Wasserdiät \\
\hline 25.-28. & 1113 & 1,6756 \% & 0,2861 & 0,0084 & $2,9 \%$ & Protogen \\
\hline 28.-29. & 1520 & 1,4098 & 0,2140 , & 0.0081 , & $3,8 \%$ & Wasserdiät \\
\hline 12. Aug. 1) & 1400 & - & 0,3698 & 0,0086 & $2,3 \%$ & 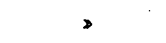 \\
\hline 12.-13. Ang. & 820 & 1,3776 & 0,3293 & 0,0074, & $2,2 \%$ & 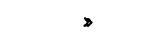 \\
\hline
\end{tabular}

Die Frage, die mir zu dem Versuch Veranlassung gab, ob nämlich der im Harn ausgeschiedene organische Phosphor ausschliesslich aus der Nahrung stammt, wird durch die vorliegenden Zahlen dahin beantwortet, dass dies nicht der Fall ist. Wir sehen, dass die organischen Phosphorverbindungen nicht aus dem Harn verschwinden, wenn auch gar keine Nahrung zugeführt wird oder die gereichte Nahrung keine organischen Phosphorverbindungeu resp. nur minimale, kaum in Betracht kommende Mengen desselben enthält. In dem vorliegenden Falle müssen die organischen Phosphorverbindungen des Harns aus dem Körper selbst stammen. Es ist dies allerdings kein Beweis, dass auch dann, wenn wir genügend Nahrung darreichen, um den Körper auf seinem Bestand zu erhalten, resp., wie dies beim Säugling nothwendig ist, um ein Wachsthum zu ermöglichen, die gleiche Menge organischer Phosphorverbindungen im Körper frei wird oder entsteht und auch zur Ausscheidung kommt, aber es ist nach dem Ergebniss der Protogenperiode wohl als wahrscheinlich anzunehmen.

Die absolute Menge des ausgeschiedenen Phosphors ist an den einzelnen Versuchstagen ziemlich constant, und zwar wird an den Hungertagen ebensoviel organischer Phosphor ausgeschieden, als an den Tagen, wo eine phosphorfreie, aber zur Erhaltung des Körpergleichgewichts genügende Nahrung zugeführt wurde. Es ist dies ein weiterer Beweis, dass die Grösse

1) Die Zahlen aus der Untersuchung der 12 stündigen Harnmenge sind verdoppelt, um sie mit den andern Zahlen leichter vergleichen $\mathrm{zu}$ können. 
des Stickstoffumsatzes auf die Ausscheidung von organischem Phosphor im Harn keinen wesentlichen Einfluss ausübt.

$\mathrm{W}^{\top}$ as das Verhältniss vom organischen Phosphor zum Gesammtphosphor im Harn anbetrifft, so unterscheiden sich die Zahlen nicht wesentlich von denen, wie wir sie bei Kuhmilchernährung beim Säugling finden, und stimmen im Allgemeinen mit denen überein, welche Oertel beim Erwachsenen gefunden hat.

Ich verzichte auf eine Besprechung jener Zahlen, die sich auf die Ausscheidung von Gesammtphosphor im Hungerzustand und bei partiellem Phosphorhunger beziehen, und will nun noch die aus diesem Versuch sich ergebenden Zahlen mit jenen Zahlen vergleichen, die ich bei demselben Kind bei Ernährung mit unverdünnter Kuhmilch gefunden hatte. Bei Zufuhr von $1,9699 \mathrm{~g} \mathrm{P}_{2} \mathrm{O}_{5}$ in der Nahrung wurden im Harn 0,851 g Gesammtphosphor und als organischer Phosphor $0,014 \mathrm{~g} \mathrm{P}_{2} \mathrm{O}_{5}$, also $2,1 \%$ des Gesammtphosphors, ausgeschieden. Wir sehen also, dass bei Zufuhr von phosphorreicher Nahrung, die auch genügend organische Phosphorverbindungen enthält, nur wenig mehr organischer Phosphor im Harn ausgeschieden wird, als bei Hungerdiät.

Meinem Versuche, während dessen das Kind gar keine Nahrung resp. keinen Phosphor in der Nahrung zugeführt erhielt, kann ich jene Versuche gegenüberstellen, in denen eine phosphorreiche Substanz als Nahrung eingeführt wird. Bei seinen Fütterungsversuchen mit Casein am Hund stellte Marcuse ${ }^{1}$ ) fest, ob Phosphor im Harn in organischer Bindung ausgeschieden wurde. Er bestimmte die Phosphorsäure im nativen Harn und in der Asche des Harns durch Titrirung mit Urannitritlösung und kam zu dem Resultat, dass die nach dem Schmelzen mit Soda und Salpeter erhaltenen Werthe stets um ein Geringes höher sind, als die der direkten Titrirung. "Die Unterschiede liegen aber dem Titrirfehler so nahe, dass er keinen sicheren positiven Schluss zu ziehen wagt », und wir dürfen wohl in Folge dessen annehmen, dass die Menge

1) Pflüg er's Archiv, Bd. 67, S. 363. 1897. 
des organischen Phosphors im Harn trotz der Zufuhr von ausschliesslich organischen Phosphorverbindungen in der Nahrung nicht wesentlich vermehrt ist. Ferner sind Fütterungsversuche mit Glycerinphosphorsäure gemacht worden. Selbst bei Zuführung grosser Mengen Glycerinphosphorsäure-Verbindungen fand $B \ddot{u} l o w^{1}$ ) nur eine sehr geringe Menge der Glycerinphosphorsäure im Harn, ebenso Pasqualis ${ }^{2}$ ) nur höchstens zweifelhafte Spuren.

Wir sehen also aus meinen Hungerversuchen einerseits und aus den Fütterungsversuchen mit Casein oder Glycerinphosphorsäure andererseits, dass auch bei den grössten Differenzen im Gehalt der Nahrung an organischen Phosphorverbindungen nur geringe Unterschiede im Gehalt des Harns an organischem Phosphor sich finden. Aus alldem ist die Thatsache zu entnehmen, dass der Theil des im Harn erscheinenden organischen Phosphors, welcher aus der Nahrung stammt, nicht bedeutend ist.

Weil bei dem Kinde, welches zu meinen Hungerversuchen und zu den Versuchen mit Protogen diente, eine Ernährungsstörung bestand, konnte ich die eine Annahme nicht vollständig ausschliessen, dass diese Störung die Ursache für das Erscheinen organischen Phosphors im Harn während des Hungerzustandes darstellte. Um dies zu entscheiden, wäre es nothwendig gewesen, bei einem gesunden Kinde im Hungerzustand den Harn zu untersuchen, wozu ich aber naturgemäss keine Gelegenheit hatte, da wir beim gesunden Kinde keine Veranlassung haben, eine Wasserdiät anzuordnen. Diese Lücke glaubte ich am besten dadurch ausfüllen zu können, dass ich an einem Erwachsenen, dessen Ernährungs- und Gesundheitszustand anscheinend normal war, einen Hungerversuch durchführte. Versuchsperson war ich selbst.

Am 13. August Abends hatte ich die letzte Mahlzeit zu mir genommen und von da an bis zum 18. August nichts als Wasser. Vom 14. August früh bis zum 18. August um

1) Plüger's Archiv, Bd. 57, S. 98.1894.

2) Annali di Chimica e farmacol. 1894. Cit. nach Maly's Jahresbericht, Bd. 24, S. 283.1895. 
dieselbe Zeit habe ich den Harn aufgesammelt. Während des Versuches habe ich von $74,7 \mathrm{~kg}$ bis $\mathrm{zu} 70,8 \mathrm{~kg}$ an Körpergewicht abgenommen. Die Untersuchung des Harns in dieser Zeit ergab folgende Werthe:

\begin{tabular}{|c|c|c|c|c|c|}
\hline \multirow[b]{2}{*}{ Datum } & \multirow[b]{2}{*}{ Harnmenge } & \multirow{2}{*}{$\begin{array}{c}\text { Menge } \\
\text { des } \\
\text { Stickstoffs }\end{array}$} & \multirow[b]{2}{*}{$\begin{array}{c}\text { Menge } \\
\text { des } \\
\text { Gesammt- } \mathrm{P}_{2} \mathrm{O}_{5}\end{array}$} & \multicolumn{2}{|c|}{ Organischer Phosphor } \\
\hline & & & & $\begin{array}{c}\text { absolute } \\
\text { Menge }\end{array}$ & $\begin{array}{c}\% \text { des } \\
\text { Gesammt- } \\
\mathrm{P}_{2} \mathrm{O}_{5}\end{array}$ \\
\hline 14.-15. Ang. & $1020 \mathrm{ccm}$. & 8,211 & 1,858 & $0,017 \mathrm{~g}$ & $0,91 \%$ \\
\hline 15.-16. & 460 & 6,7459 & 1,9016 & 0,0294 . & $1,5 \%$ \\
\hline 16.-17. & 1220 & 7,9117 & 2,4393 , & 0,0344 . & $1,4 \%$ \\
\hline 17.-18., & 1540 & 11,4807 & 2,5265 , & 0,0573 , & $2,3 \%$ \\
\hline
\end{tabular}

Demnach wird auch vom gesunden Erwachsenen im Hungerzustand organischer Phosphor ausgeschieden, der wohl zum Theil aus Zerfall von Körpersubstanz, zum Theil aus Secreten herrührt.

In Betreff der Ausscheidung von Gesammtphosphor und organischem Phosphor im Harn stimmen die Ergebnisse meines Hungerversuches im Grossen und Ganzen mit den Zahlen überein, die Oertel für den Erwachsenen bei gemischter Diät gefunden hat. $\mathrm{W}^{\top}$ enn auch die Menge des Stickstoffs im Harn niedriger ist als unter normalen Verhältnissen, so wird doch während des Hungerzustandes im Durchschnitt ebensoviel Phosphorsäure ausgeschieden, als bei ausreichender Ernährung. Auch das Verhältniss vom organischem Phosphor zum Gesammtphosphor im Harn ändert sich nur wenig; es erscheint auffallend, dass gerade am ersten Hungertage die niedrigsten Zahlen in Betreff der Ausscheidung des organischen Phosphors sich finden. Wenn die 4 Tage eine genügend lange Versuchsperiode darstellen, um irgend welche Schlüsse zu erlauben, so würden sie darauf hindeuten, dass während einer Hungerperiode die Ausscheidung organischen Phosphors im Harn ansteigt, und zwar schneller als die Gesammtphosphorsäure, von der wir bereits wissen, dass während des Hungerns nicht weniger ausgeschieden wird, als während ausreichender Ernährung. 
Wenn wir uns fragen, aus welchen Organen oder Geweben die organischen Phosphorverbindungen stammen, die während des Hungerzustandes im Harn ausgeschieden werden, so können uns dafür Untersuchungen, wie sie z. B. von Chossat, C. Voit, S. Munk und zuletzt von Weiske ausgeführt wurden, einen Anhaltspunkt geben, jene Untersuchungen, bei denen festgestellt wurde, in welcher Weise die verschiedenen Organe des Thierkörpers sich an dem beim Hunger eintretenden Körperverluste betheiligen und wie sich die $\mathrm{Zu}$ sammensetzung der betreffenden Organe ändert. Wir wissen, dass das an Phosphaten reiche. Skelett auch bei lange dauerndem Hunger nur unbedeutend an Gewicht verliert und am Schluss des Hungerversuchs noch ungefähr denselben Gehalt an Phosphor aufweist, wie am Anfang desselben.

Bekannt ist ausserdem aus den Versuchen von Chossat, Bibra u. A., dass das Gehirn und Rückenmark bei Inanition nicht abnimmt resp. dass demselben auf Kosten anderer Organe Ersatz geliefert wird.

Dagegen betheiligen sich an den beim Hunger eintretenden Körperverluste hauptsächlich Milz, Leber, Lunge, Därme etc., gerade jene Organe, die durch einen hohen Gehalt an Nuclein ausgezeichnet sind.

Ist unter diesen Umständen der Schluss unberechtigt, dass gerade der Zerfall der nucleinreichen Organe die Quelle des im Harn erscheinenden organischen Phosphors im Hungerzustand ist, wenn wir noch berücksichtigen, dass die organische Phosphorverbindung, welche hauptsächlich im Gehirn und Rückenmark enthalten ist, nämlich das Lecithin, leichter verbrennbar ist, als die übrigen organischen Phosphorverbindungen?

Zuletzt will ich noch auf die Frage eingehen, in wie weit der Gehalt des Harns an organischen Phosphorverbindungen ein Mittel zur Prüfung der Oxydationsfähigkeit des betreffenden Organismus sein könnte. Aus meinen Untersuchungen ergibt sich das Resultat, dass der im Harn ausgeschiedene organische Phosphor zum Theil aus der Nahrung stammt, zum Theil aus dem Abbau von Körpersubstanz oder aus Secreten der verschiedenen Organe. Wenn wir diese 
beiden Theile trennen könnten, dann würde die Grösse der Ausscheidung des aus der Nahrung stammenden Phosphors einen Maassstab für die Oxydationskraft des Organismus darstellen, vorausgesetzt, dass wir verschiedenen Kindern gleiche Mengen von bestimmten organischen Phosphorverbindungen in der Nahrung zuführen würden.

Was den zweiten Theil des organischen Phosphors im Harn, den aus dem Körper stammenden anbetrifft, so liegt bisher kein Beweis vor, dass dessen Grösse von der Oxydationsfähigkeit des Organismús abhängt. Im Gegentheil scheint mir das Resultat des Hungerversuches, den ich an mir selbst ausgeführt habe, nur darauf hinzudeuten, dass im Körper organische Phosphorverbindungen entstehen oder frei werden, die ausgeschieden werden, bevor sie noch verbrannt werden können. 


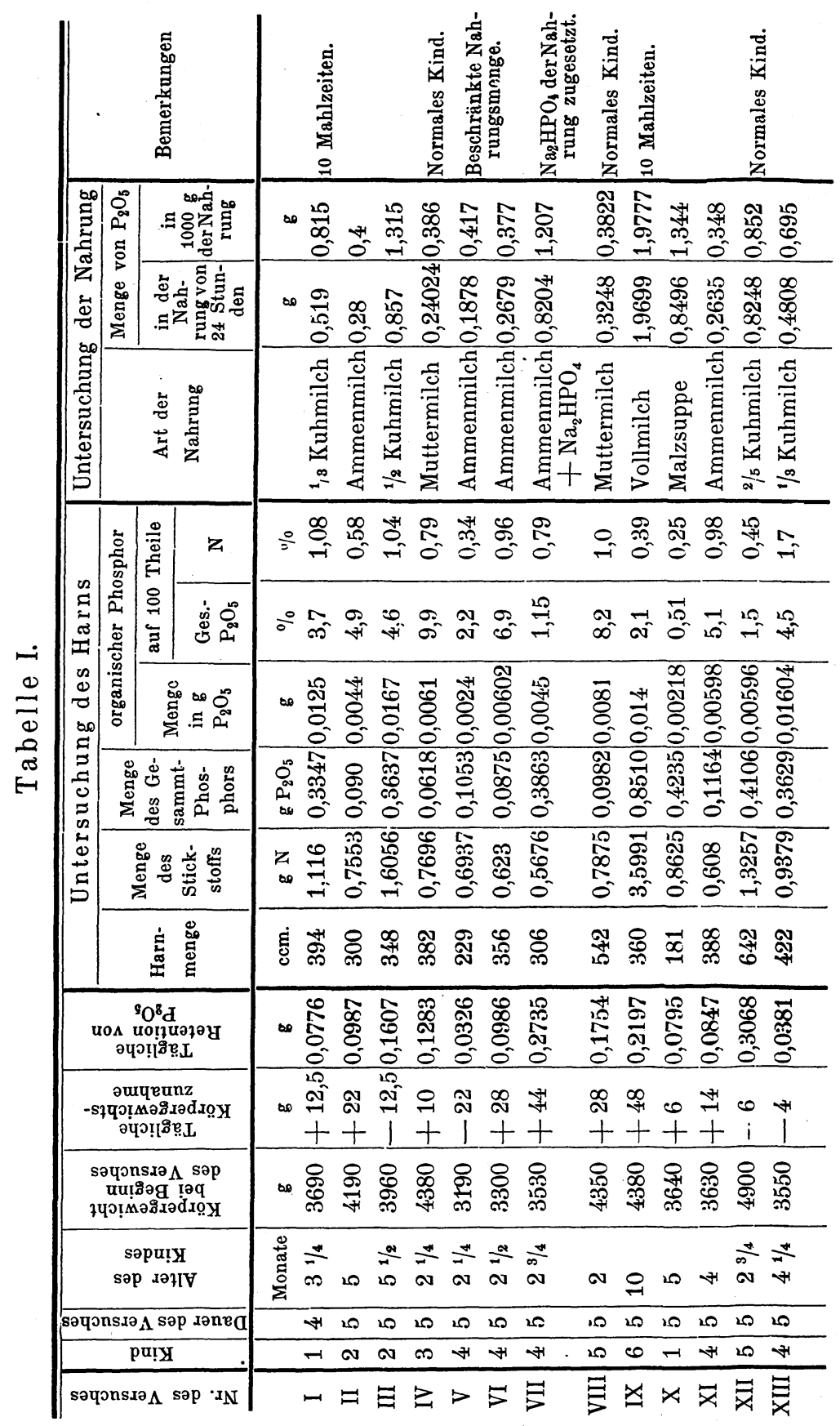




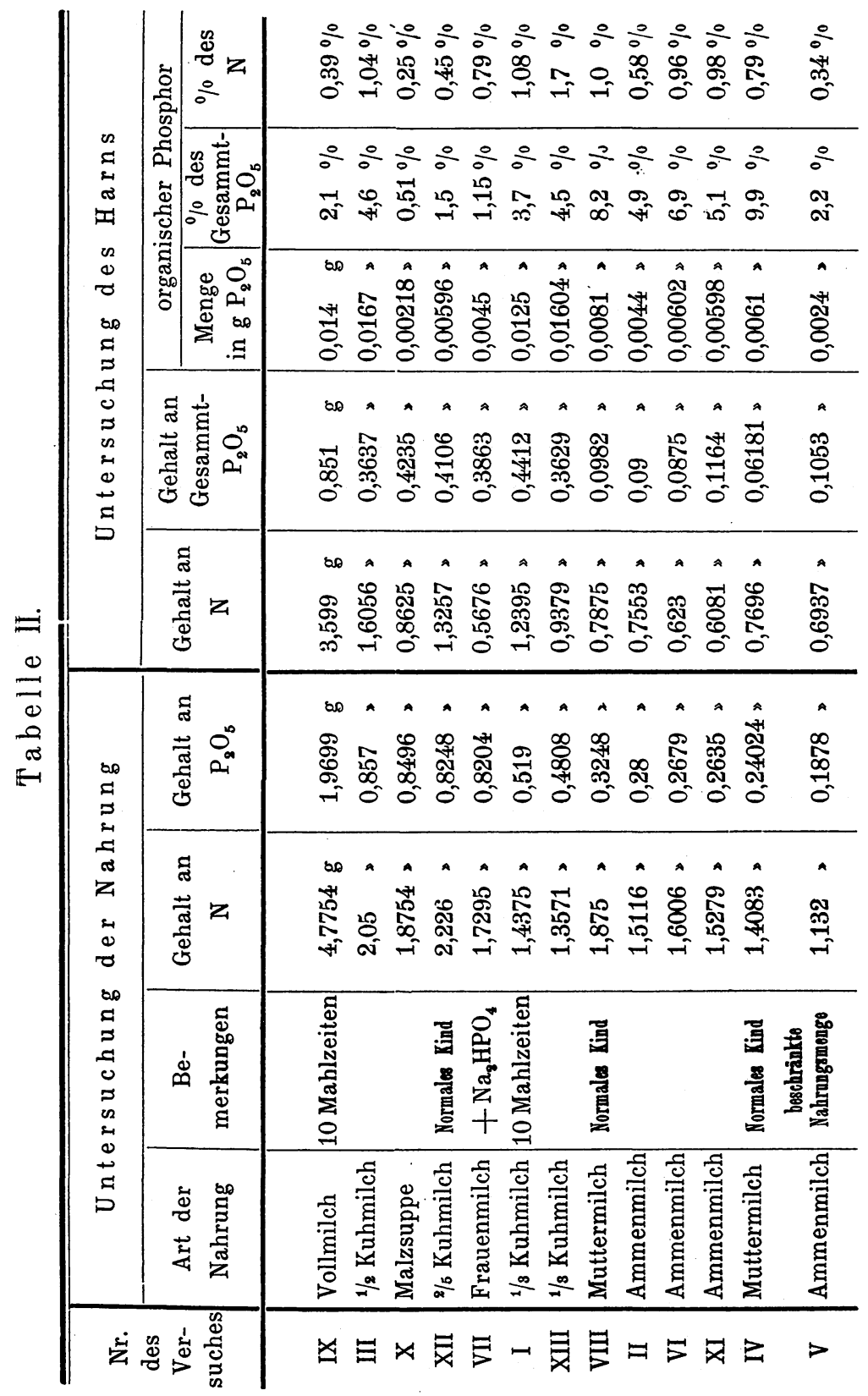

Hoppe-Seyler's Zeitschrift f. physiol. Chemie. XXIX. 


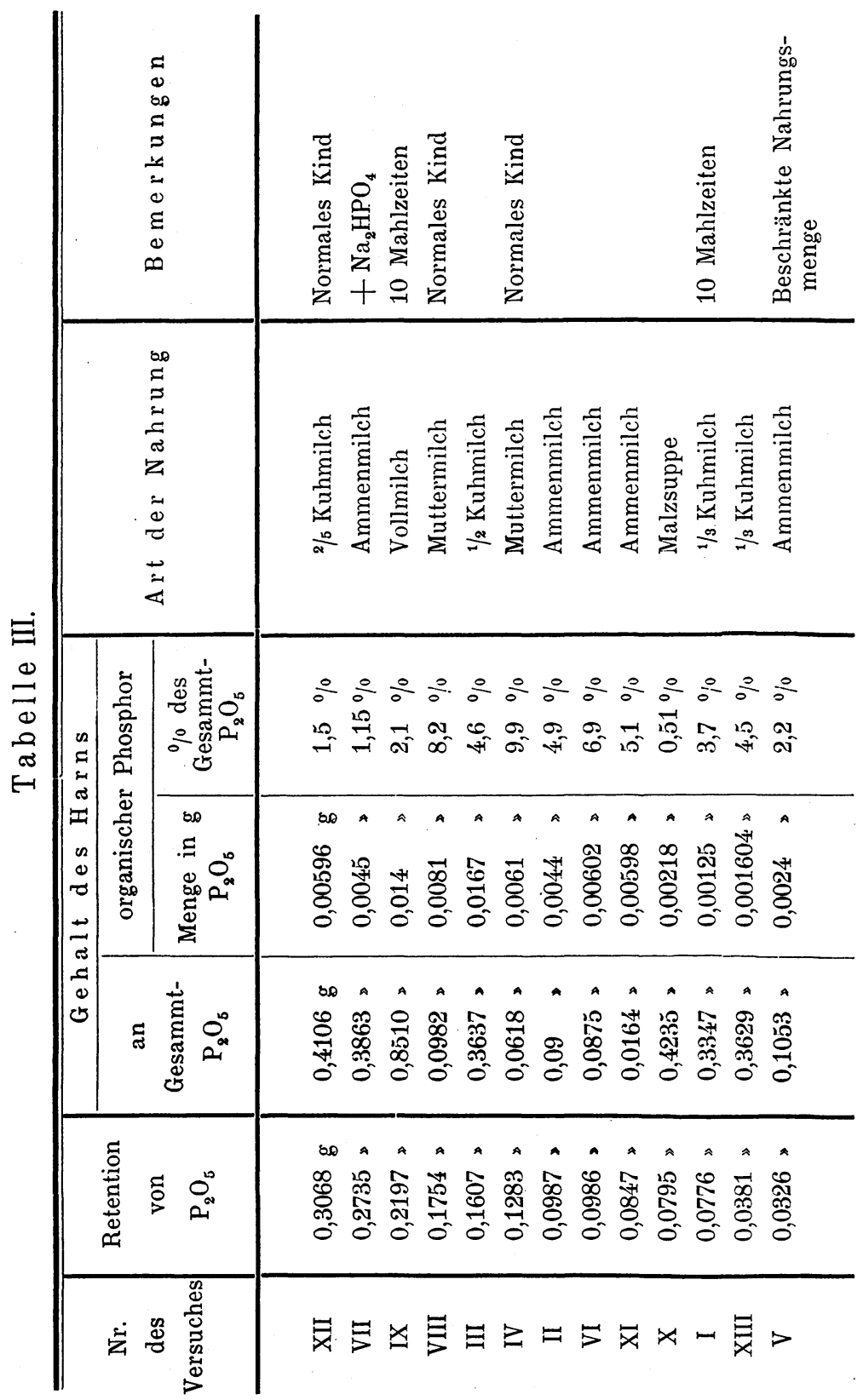




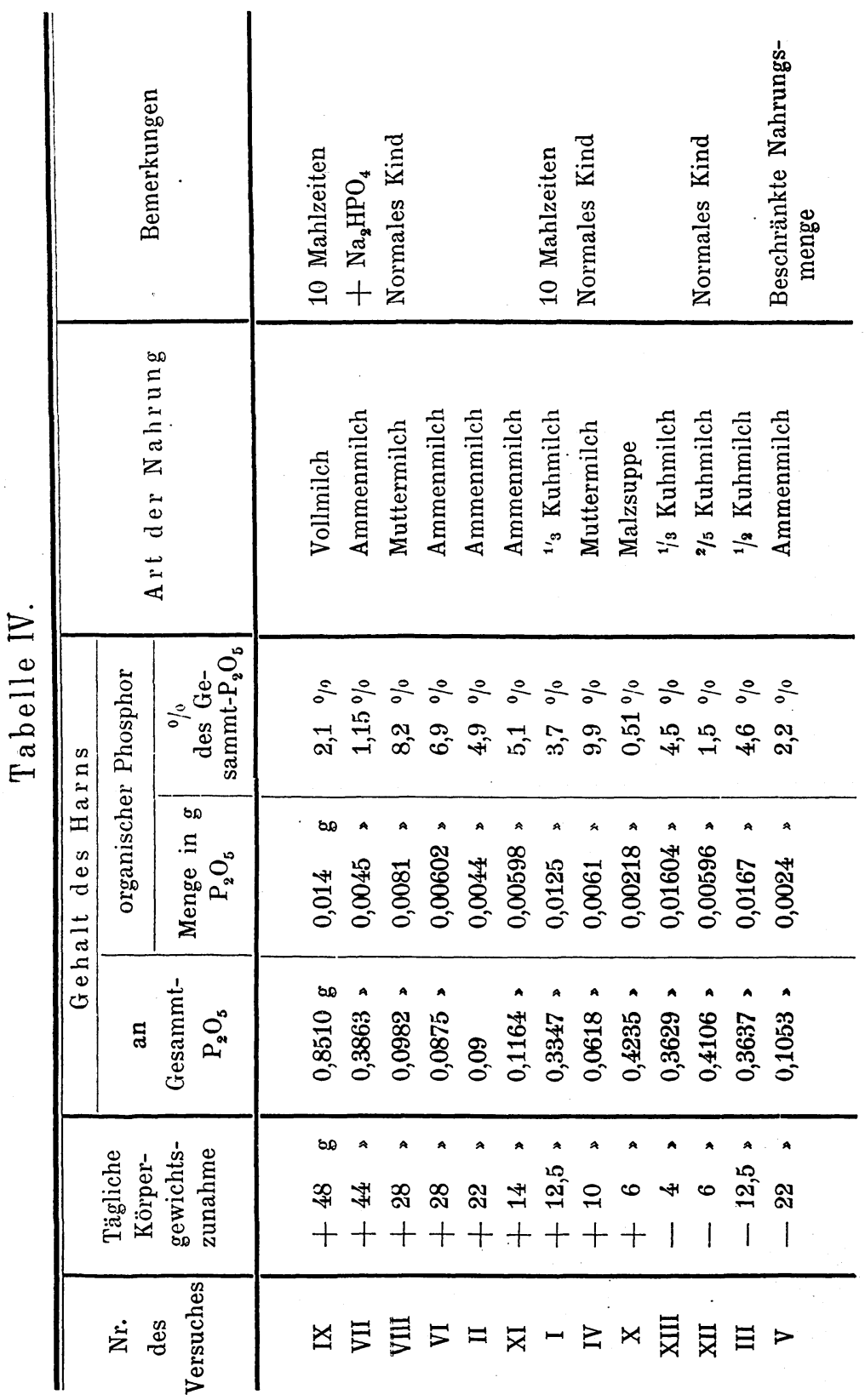




\begin{tabular}{|c|c|c|c|}
\hline \multirow{4}{*}{ 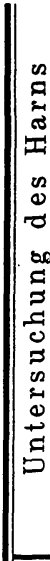 } & \multirow{2}{*}{ 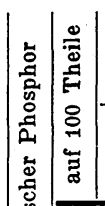 } & 离 & 薄 \\
\hline & & 离歒 & 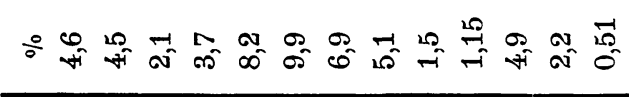 \\
\hline & & 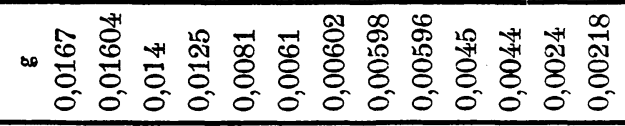 \\
\hline & \multicolumn{2}{|c|}{ 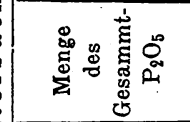 } & 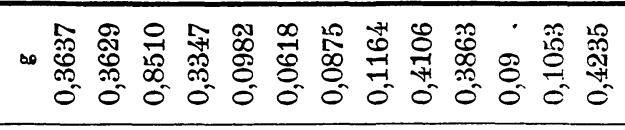 \\
\hline & \multicolumn{2}{|c|}{ 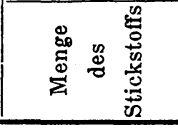 } & 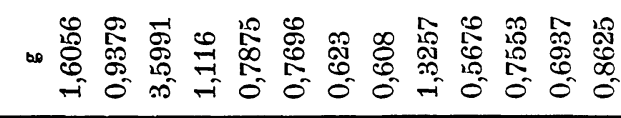 \\
\hline \multicolumn{3}{|c|}{ 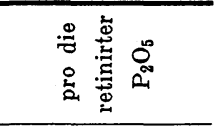 } & 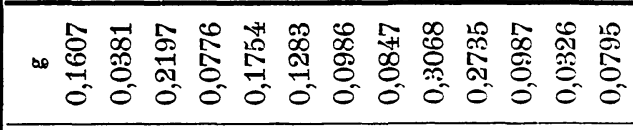 \\
\hline \multicolumn{3}{|c|}{ 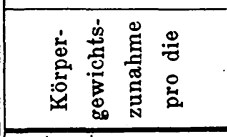 } & 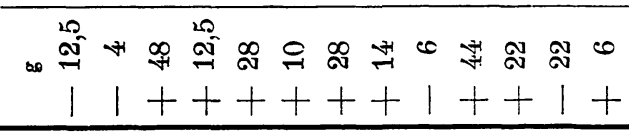 \\
\hline \multirow{2}{*}{\multicolumn{2}{|c|}{ 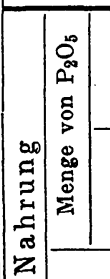 }} & 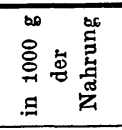 & 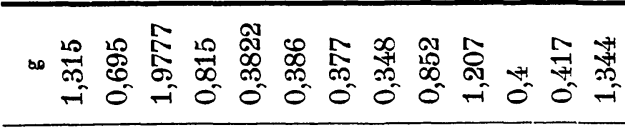 \\
\hline & & & 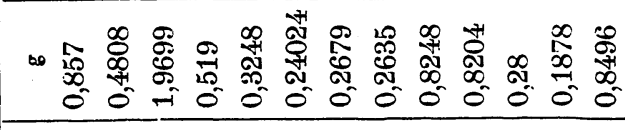 \\
\hline \multirow[t]{2}{*}{ 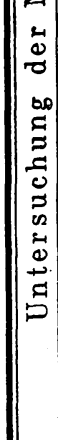 } & \multicolumn{2}{|c|}{ 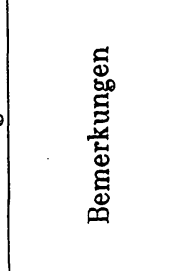 } & 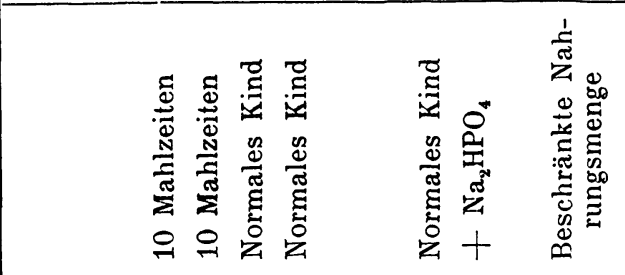 \\
\hline & \multicolumn{2}{|c|}{ 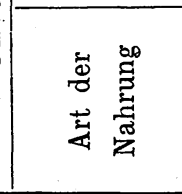 } & 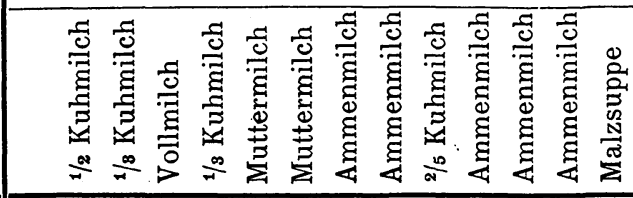 \\
\hline \multicolumn{3}{|c|}{ pui:Y } & 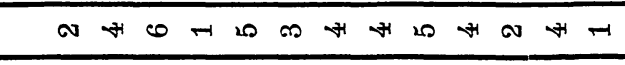 \\
\hline \multicolumn{3}{|c|}{ 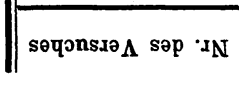 } & 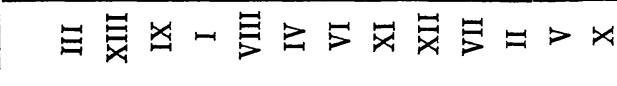 \\
\hline
\end{tabular}




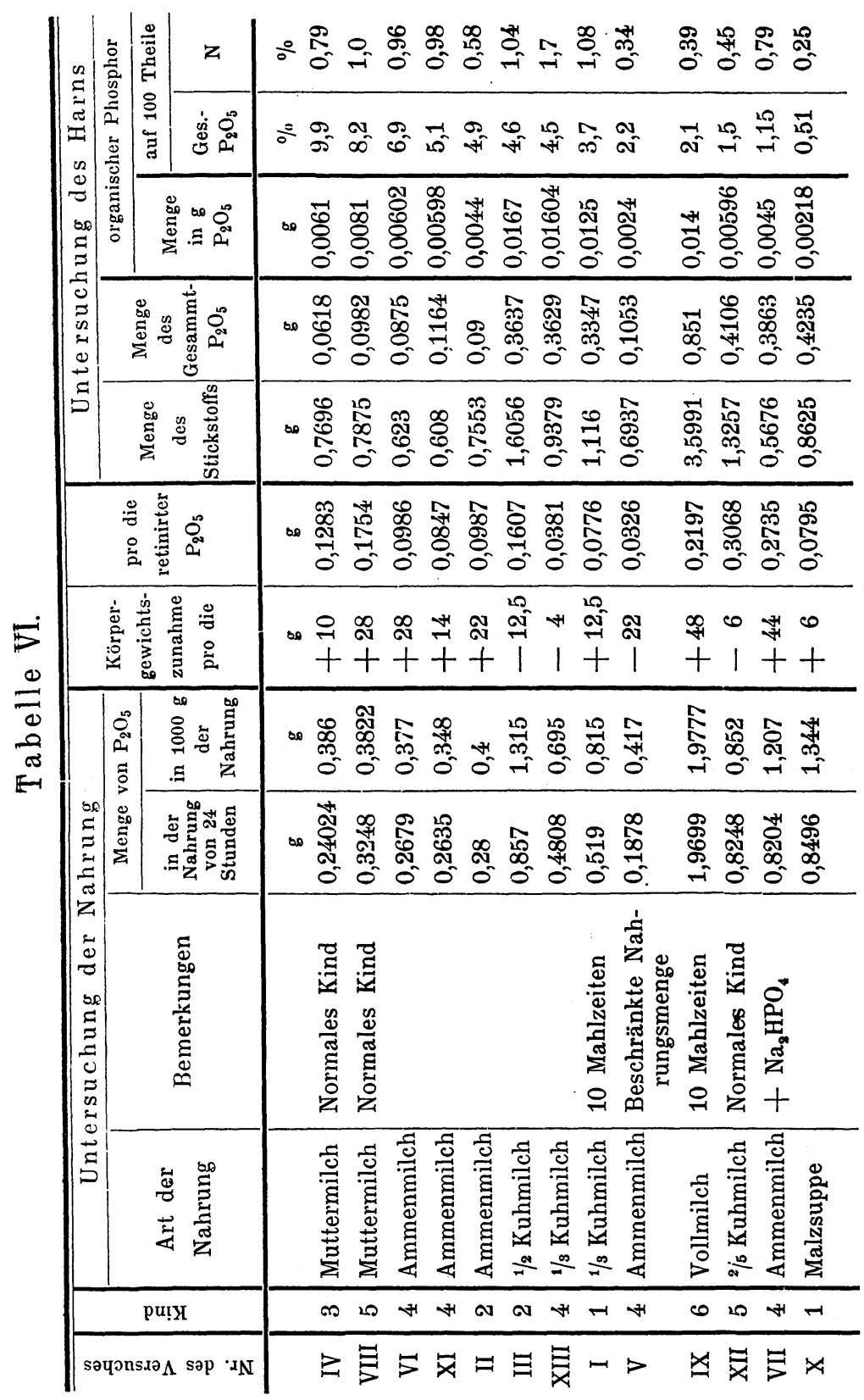




\section{Krankengeschichten.}

\section{Kind 1 .}

Die ersten 14 Tage an der Brust, dann mit 1/3 Kuhmilch in 2 stündigen Pausen ernährt, wurde am 24. April 1899-11 Wochen alt - in die Poliklinik gebracht, weil nach Angabe der Pflegefrau das Kind seit einigen Tagen sehr unruhig war, weniger trank als vorher und häufig erbrach.

Ziemlich mageres blasses Kind von 4100 g Körpergewicht. Zeichen von beginnender Rachitis, Bronchitis, Hernia inguinalis dextra.

Nach der Aufnahme in die stationäre Abtheilung erhält das Kind auch weiterhin 2 stündlich $1 / 3 \mathrm{Kuhmilch}$, von der es zuerst 1000 bis $900 \mathrm{~g}$, später weniger trinkt. In den ersten Tagen bestehendes Erbrechen verschwindet. Dabei nahm das Kind bis zum 10. Mai etwa $300 \mathrm{~g}$ an Körpergewicht ab. An diesem Tage wird Versuch I begonnen. Wenige Tage nach Beendigung des Versuches traten Erscheinungen von Seiten des Magendarmkanals auf, die uns veranlassten, das Kind weiterhin mit Frauenmilch zu ernähren. Dabei besserte sich langsam das Allgemeinbefinden, sowie die Verdauungsstörung, die Körgergewichtskurve ging zwar bis Ende Mai noch weiter herunter (29. Mai $3160 \mathrm{~g}$ ), dann aber blieb das Körpergewicht einige Zeit zwischen 3100 und $3200 \mathrm{~g}$ stehen. Vom 19. Juni an erhielt das Kind Malzsuppe und es trat unter dem Einfluss der Ernährung gleichzeitig mit dem weiteren Fortschreiten der Besserung ${ }^{\circ}$ im Allgemeinbefinden und im Ernährungszustand regelmässige Körpergewichtszunahme ein.

Am 11. Juli (Körpergewicht $3640 \mathrm{~g}$ ) wurde ein Stoffwechselversuch (Nr. X) begonnen, während dessen das Kind täglich 631,5 g Malzsuppe in 5 Mahlzeiten erhielt.

\section{Kind 2 .}

Wurde am 15. März 1899 - 1/4 Jahr alt - in die Poliklinik gebracht. Von der Pflegefrau, welche erst kurze Zeit das Kind in Pflege hat, ist betreffs der Vorgeschichte nur soviel zu erfahren, dass Magendarmstörungen nicht bestanden haben sollen und dass das Kind seit einiger Zeit huste. Als Nahrung wurde verabreicht 1/3 Milch mit 2/s Haferschleim. 
Das ziemlich magere, damals $3840 \mathrm{~g}$ schwere Kind, bei dem eine Bronchitis constatirt wurde, blieb bei derselben Ernährung und nahm bis zum 3. Mai $1 \mathrm{~kg}$ an Körpergewicht zu. Es wurde an diesem Tage in die stationäre Abtheilung aufgenommen und vom 12. Mai an, da in der Zwischenzeit Magendarmstörungen aufgetreten waren, mit Frauenmilch ernährt. Bei dieser Ernährung wurde Versuch II durchgeführt. Nach dem Versuch erhielt das Kind 1/3 Kuhmilch, später 1/2 Kuhmilch.

Am 30. Mai wurde Versuch III begonnen, während dessen das Kind täglich $360 \mathrm{~g}$ Kuhmilch mit $290 \mathrm{~g}$ Wasser verdünnt als Nahrung erhielt.

Kind 3 .

Am 30. März 1899 als erstes Kind von gesunden Eltern geboren, mit einem Körpergewicht von $2920 \mathrm{~g}$. Brustkind. Am 4. Tage hatte das Kind bis zu 2650 g abgenommen, von da an ging die Körpergewichtskurve mit nur sehr geringen Schwankungen regelmässig in die Höhe. Am 9. Tage ist das Anfangsgewicht erreicht, am 1. Mai $3620 \mathrm{~g}$, am 1. Juni $4400 \mathrm{~g}$. Anzahl und Aussehen der Stühle sind normal, nie Zeichen einer Magendarmerkrankung, ausgenommen die eine Beobachtung, dass das Kind, wenn es nach dem Trinken auf die Waagschale gelegt wird, hin und wieder geringe Mengen von Nahrung aus dem Mund zurückfliessen lässt. Vom 6. bis zum 11. Juni Stoffwechselversuch (IV). Das Verhalten des Kindes in der späteren Zeit, seine weitere Entwicklung und körperliche Zunahme zeigt uns, dass wir es mit einem anscheinend g e sunden Brustkinde zu thun haben.

Während des Versuches erhält das Kind 5 Mahlzeiten, trinkt jedesmal an beiden Brüsten. Die Menge der getrunkenen Nahrung wird durch Wägung des Kindes vor und nach dem Anlegen bestimmt. Zur Untersuchung der Nahrung werden vor und nach der Mahlzeit Proben der Milch aus der Brust entnommen.

\section{$\mathrm{K}$ ind 4.}

Erstes Kind gesunder Eltern, wurde in den ersten 10 Tagen an der Brust, dann mit $1 / \mathbf{3}$ Kuhmilch in 3 stündlichen Pausen ernährt. Am 1. Juni wurde das 8 Wochen alte Kind von der Mutter mit der Angabe in die Poliklinik gebracht, dass es vor etwa 10 Tagen erkrankt sei. Die Stühle seien wässerig, Nahrung würde öfter verweigert und das Kind sei im Körpergewicht heruntergekommen.

Das Kind, welches bei der Aufnahme $3130 \mathrm{~g}$ wog, war ziemlich stark abgemagert und blass. An den inneren Organen nichts Abnormes. Bei Wasserdiät nimmt das Kind in 24 Stunden $100 \mathrm{~g}$ an Körpergewicht ab, um dann bei Ernährung mit Frauenmilch mehr und mehr zuzunehmen. In dieser Zeit regelmässiger Zunahme wurden die Versuche V, VI und VII durchgeführt und zwar die beiden ersten bei Ernährung mit Frauenmilch, beim 3. Versuch wurden der Frauenmilch Natriumphosphate zugesetzt. In der späteren Zeit, etwa von Anfang Juli an, traten häufig 
wiederum neue Erkrankungen ein, deren acute Erscheinungen zwar stets bald verschwanden, ' die aber doch der Grund waren, dass das Kind während der nächsten Wochen an Körpergewicht nicht zunahm. In dieser Zeit wurde bei Ernährung mit Frauenmilch Versuch XI und bei Ernährung mit verdünnter Kuhmilch Versuch XIII durchgeführt.

\section{$\mathrm{K}$ ind 5.}

Am 6. Mai 1899 mit einem Körpergewicht von $2870 \mathrm{~g}$ geboren, hereditär nicht belastet, von Anfang an der Brust ernährt. Nach der physiologischen Körpergewichtsabnahme hatte das Kind am 9. Lebens- . tage sein Anfangsgewicht wieder erreicht und nahm von da an regelmässig zu. Mit Ausnahme einer kurzen Periode von etwa 8 Tagen, in der das Kind Zeichen einer leichten Magendarmerkrankung zeigte, war das Allgemeinbefinden des Kindes stets ungestört. Am Ende des ersten Lebensmonats wog das Kind $3420 \mathrm{~g}$, am Ende des zweiten Monats $4400 \mathrm{~g}$, am Ende des 3 . Monats $5160 \mathrm{~g}$. Die weitere Entwicklung des Kindes und seine Körpergewichtszunahme entsprach normalen Verhältnissen. Es wurde bei dem Kinde zuerst ein Versuch (VIII) bei Ernährung mit Frauenmilch gemacht und 3 Wochen später ein Versuch XII bei Ernährung mit 2/5 Kuhmilch.

\section{$\mathrm{K}$ ind 6 .}

Wurde am 20. Januar 1899, 3 Monate alt, in unsere Poliklinik gebracht. Der Vater soll an Schwindsucht gestorben sein. Das Kind wurde von Anfang an künstlich ernährt und zwar in 2 stündlichen Pausen mit verdünnter Kuhmilch. Wegen starker Diarrhöe musste diese Ernährung nach 4 Wochen aufgegeben werden, das Kind erhielt von da an Hafergrütze mit Milch. In der letzten Zeit häufiges starkes Erbrechen.

Das schlecht genährte Kind mit $3370 \mathrm{~g}$ Körpergewicht, an dem ausser Zeichen von Rachitis nichts Abnormes nachweisbar war, wurde mit verdünnter Malzsuppe ernährt, erholte sich dabei zusehends und nahm im Laufe von 5 Wochen um etwa $600 \mathrm{~g}$ an Körpergewicht zu. Dann erkrankte es von Neuem an einer Magendarmstörung, nahm bis $3300 \mathrm{~g}$ an Körpergewicht ab, um dann im Laufe der Monate April und Mai langsam bis zu $3900 \mathrm{~g}$ zuzunehmen. Im Laufe des Monats Juni wurde das Kind nie vorgezeigt, erst am 10. Juli erschien die Mutter in der Poliklinik. Nach ihrer Angabe hat das Kind in der Zwischenzeit täglich etwa 1 Liter unverdünnte Kuhmilch in 1-2 stündlichen Pausen getrunken.

Das mittlerweile 10 Monate alte Kind von $4300 \mathrm{~g}$ Körpergewicht war sehr blass, am Skelett Zeichen von Rachitis, an den inneren Organen nichts Abnormes nachweisbar. Nach der Aufnahme in die Klinik wurde bei derselben Ernährung, d. h. bei Darreichung von Vollmilch in 2stïndlichen Pausen ein Stoffwechselversuch durchgeführt. Da das Kind in den nächsten Tagen nach dem Versuch häufig erbrach und ausserdem an Körpergewicht abnahm, wurde am 23. Juli die Nahrung ausgesetzt. 


\section{Beläge.}

Versuch I.

\begin{tabular}{|c|c|c|c|c|c|}
\hline \multirow{2}{*}{ Datum } & \multirow{2}{*}{ Harnmenge } & \multirow{2}{*}{$\begin{array}{c}\mathrm{N} \\
\text { in } 10 \mathrm{ccm} .\end{array}$} & \multirow{2}{*}{$\begin{array}{c}\left.\mathrm{P}_{2} \mathrm{O}_{5}{ }^{1}\right) \\
\text { in } 50 \mathrm{ccm} .\end{array}$} & \multicolumn{2}{|c|}{$\begin{array}{l}\text { pro die werden aus- } \\
\text { geschieden }\end{array}$} \\
\hline & & & & $\mathbf{N}$ & $\mathrm{P}_{2} \mathrm{O}_{5}$ \\
\hline 11.V. 1899 & $330 \mathrm{ccm}$. & $31,15 \mathrm{mg}$ & $46,66 \mathrm{mg}$ & $1,0279 \mathrm{~g}$ & $0,3079 \mathrm{~g}$ \\
\hline 12.V. 1899 & $37 \check{\jmath}$ & 28,0 & 42,04 & 1,050 & 0,3156 \\
\hline 13. V.1899 & 475 & $27 ; 3$ & 37,19 & 1,2967 & 0,3533 . \\
\hline \multirow[t]{2}{*}{ 14. V. 1899} & 395 & \multirow[t]{2}{*}{28,35} & \multirow[t]{2}{*}{38,42 , } & 1,0892 & 0,3035 \\
\hline & $1575 \mathrm{ccm}$. & & & $4,4639 \mathrm{~g}$ & $1,2800 \mathrm{~g}$ \\
\hline
\end{tabular}

Vom Mischharn der ganzen Periode werden zu den Wägebestimmungen des Phosphors je $50 \mathrm{ccm}$. verwendet. Bei vorhergehender Verbrennung der organischen Substanzen nach Neumann finden sich:

A. Gesammtphosphor in $50 \mathrm{ccm}$. I. $0,0666 \mathrm{~g}$, II. $0,0664 \mathrm{~g}$, Mittel $0,0665 \mathrm{~g}^{\prime} \mathrm{Mg}_{2} \mathrm{P}_{2} \mathrm{O}_{7}=0,0425 \mathrm{~g} \mathrm{P}_{2} \mathrm{O}_{5} ;$ demnach in $1575 \mathrm{ccm}$. Harn: $1,3387 \mathrm{~g} \mathrm{P}_{2} \mathrm{O}_{5}$.

B. Direkte Fällung mit Molybdänsäure (anorganische Phosphate) in $50 \mathrm{ccm}$. I. $0,0639 \mathrm{~g}$, II. $0,0641 \mathrm{~g}$, Mittel 0,064 g $\mathrm{Mg}_{\mathbf{z}} \mathrm{P}_{\mathbf{z}} \mathrm{O}_{7}$ $=0,0409 \mathrm{~g} \mathrm{P}_{2} \mathrm{O}_{5}$; demnach in $1575 \mathrm{ccm}$. Harn $1,2694 \mathrm{~g}_{2} \mathrm{O}_{5}$.

C. Organischer Phosphor in $1575 \mathrm{ccm}$. Harn: 0,0499 $\mathrm{g}_{2} \mathrm{O}_{5}$.

Versuch II.

\begin{tabular}{|c|c|c|c|c|c|}
\hline \multirow{2}{*}{ Datum } & \multirow{2}{*}{ Harnmenge } & \multirow{2}{*}{$\begin{array}{c}\mathrm{N} \\
\text { in } 10 \mathrm{ccm} .\end{array}$} & \multirow{2}{*}{$\begin{array}{c}\left.\mathrm{P}_{2} \mathrm{O}_{5}{ }^{2}\right) \\
\text { in } 50 \mathrm{ccm} .\end{array}$} & \multicolumn{2}{|c|}{$\begin{array}{l}\text { pro die werden aus- } \\
\text { geschieden }\end{array}$} \\
\hline & & & & $\mathbf{N}$ & $\mathrm{P}_{2} \mathrm{O}_{5}$ \\
\hline 16. V. 1899 & $420 \mathrm{ccm}$. & $19,95 \mathrm{mg}$ & $7,21 \mathrm{mg}$ & $0,8379 \mathrm{~g}$ & $0,0606 \mathrm{~g}$ \\
\hline 17. V. 1899 & 335 » & $22,4 \quad \gg$ & 11,78 & 0,7504 & 0,0789 । \\
\hline 18. V.1899 & 240 & 26,25 & 17,32 & 0,630 & 0,0831 \\
\hline 19.V. 1899 & 285 & 27,65 & 18,25 & 0,788 & 0,1040 » \\
\hline \multirow[t]{2}{*}{ 20. V. 1899} & 220 & 35,0 & 22,64 & 0,770 & 0,0996 \\
\hline & $1500 \mathrm{ccm}$. & & & $3,7763 \mathrm{~g}$ & $0,4262 \mathrm{~g}$ \\
\hline
\end{tabular}

1) Durch Titration mit Urannitratlösung bestimmt.

2) Durch Titration bestimmt. 
Mischharn.

A. Gesammtphosphor in $100 \mathrm{ccm}$. I. $0,0467 \mathrm{~g}$, II. $0,0471 \mathrm{~g}$, Mittel 0,0469 g $\mathrm{Mg}_{2} \mathrm{P}_{2} \mathrm{O}_{7}=0,029997 \mathrm{~g} \mathrm{P}_{2} \mathrm{O}_{5}$ demnach in $1500 \mathrm{ccm}$. $0,4499 \mathrm{~g} \mathrm{P}_{2} \mathrm{O}_{5}$.

B. Anorganische Phosphate. Die Wägebestimmungen gehen verloren. Die direkte Titration von $50 \mathrm{ccm}$. des Mischharns ergibt $0,014322 \mathrm{~g} \mathrm{P}_{2} \mathrm{O}_{5}$, das sind in $1500 \mathrm{ccm}$. 0,42966 g $\mathrm{P}_{2} \mathrm{O}_{5}$. Bei der Titration der einzelnen Tagesmengen hatte ich gefunden in $1500 \mathrm{ccm}$. Harn $0,4262 \mathrm{~g} \mathrm{P}_{2} \mathrm{O}_{5}$. Als Mittelwerth ergibt sich also $0,4279 \mathrm{~g} \mathrm{P}_{2} \mathrm{O}_{5}$.

C. Organischer Phosphor in $1500 \mathrm{ccm}$. Harn $0,022 \mathrm{~g}_{2} \mathrm{O}_{5}$.

Versuch III.

\begin{tabular}{|c|c|c|c|c|c|}
\hline \multirow{2}{*}{ Datum } & \multirow{2}{*}{ Harnmenge } & \multirow{2}{*}{$\begin{array}{c}\mathrm{N} \\
\text { in } 10 \mathrm{ccm} \text {. }\end{array}$} & \multirow{2}{*}{$\begin{array}{c}\mathrm{P}_{2} \mathrm{O}_{5} \\
\text { in } 50 \mathrm{ccm} .\end{array}$} & \multicolumn{2}{|c|}{$\begin{array}{l}\text { pro die werden aus- } \\
\text { geschieden }\end{array}$} \\
\hline & & & & $\mathrm{N}$ & $\mathrm{P}_{2} \mathrm{O}_{5}$ \\
\hline 30. V. 1899 & $400 \mathrm{ccm}$. & $36,05 \mathrm{mg}$ & $34,419 \mathrm{mg}$ & $1,442 \mathrm{~g}$ & $0,27 \check{3} 3 \mathrm{~g}$ \\
\hline 31. V. 1899 & $36 \check{~}$ & $43,0 \check{0} \gg$ & 50,47 & $1,5713 »$ & 0,3684 » \\
\hline 1.VI.1899 & 300 & 48,3 & 55,902 & 1,449 & 0,3354 » \\
\hline 2.VI.1899 & 335 & 56,0 & 62,139 & 1,8766 ১ & 0,4163 \\
\hline \multirow[t]{2}{*}{ 3.VI.1899 } & 340 & \multirow[t]{2}{*}{49,7} & \multirow[t]{2}{*}{53,13} & 1,6898 & 0,3379 » \\
\hline & $1740 \mathrm{ccm}$. & & & $8,0281 \mathrm{~g}$ & $1,7333 \mathrm{~g}$ \\
\hline
\end{tabular}

A. Gesammtphosphor in $50 \mathrm{ccm}$. $1.0,0813 \mathrm{~g}$, II. $0,0821 \mathrm{~g}$, Mittel $0,0817 \mathrm{~g}_{\mathrm{Mg}_{2}} \mathrm{P}_{2} \mathrm{O}_{7}=0,052255 \mathrm{~g} \mathrm{P}_{2} \mathrm{O}_{5}$; demnach in $1740 \mathrm{ccm}$. $1,8185 \mathrm{~g}_{2} \mathrm{O}_{5}$.

B. Anorganische Phosphate: I. 0,0777 g, II. $0,0782 \mathrm{~g}$, Mittel $0,07795 \mathrm{~g} \mathrm{Mg}_{\mathbf{x}} \mathrm{P}_{2} \mathrm{O}_{7}=0,04986 \mathrm{~g} \mathrm{P}_{2} \mathrm{O}_{5}$; demnach in $1740 \mathrm{ccm}$. $1,7351 \mathrm{~g} \mathrm{P}_{2} \mathrm{O}_{5}$.

C. Organischer Phosphor in $1740 \mathrm{ccm}$. $-1,7351 \mathrm{~g} \mathrm{P}_{2} \mathrm{O}_{6}$.

Versuch IV.

\begin{tabular}{|c|c|c|c|c|c|}
\hline \multirow{2}{*}{ Datum } & \multirow{2}{*}{ Harnmenge } & \multirow{2}{*}{$\begin{array}{c}\mathrm{N} \\
\text { in } 10 \mathrm{ccm} .\end{array}$} & \multirow{2}{*}{$\begin{array}{c}\mathrm{P}_{2} \mathrm{O}_{5} \\
\text { in } 50 \mathrm{ccm} .\end{array}$} & \multicolumn{2}{|c|}{$\begin{array}{c}\text { pro die werden aus- } \\
\text { geschieden }\end{array}$} \\
\hline & & & & $\mathrm{N}$ & $\mathrm{P}_{2} \mathrm{O}_{5}$ \\
\hline 7.VI.1899 & $300 \mathrm{ccm}$ & $23,4 \check{\mathrm{mg}}$ & $6,77 \quad \mathrm{mg}$ & $0,7035 \mathrm{~g}$ & $0,0406 \mathrm{~g}$ \\
\hline 8.VI.1899 & 365 & 22,75 & 8,0625 & 0,83037 & 0,0589 \\
\hline 9.VI.1899 & 425 & 18,9 & 6.77 & ه & 0,05575 । \\
\hline 10.VI.1899 & 375 & 19,95 & 8,385 & 0,7481 & $0,0629 »$. \\
\hline \multirow[t]{2}{*}{ 11.VI.1899 } & 445 & \multirow[t]{2}{*}{ 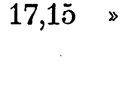 } & \multirow[t]{2}{*}{$6,1275 \gg$} & 0.7632 & 0,0545 \\
\hline & $1910 \mathrm{ccm}$. & & & $3,8484 \mathrm{~g}$ & $0,2745 \mathrm{~g}$ \\
\hline
\end{tabular}


Mischharn.

A. Gesammtphosphor in $100 \mathrm{ccm}$. I. $0,0251 \mathrm{~g}$, II. $0,0255 \mathrm{~g}$, Mittel $0,0253 \mathrm{~g} \mathrm{Mg}_{2} \mathrm{P}_{2} \mathrm{O}_{7}=0,01618 \mathrm{~g} \mathrm{P}_{2} \mathrm{O}_{5}$; demnach in $1910 \mathrm{ccm}$. $=0,30904 \mathrm{~g} \mathrm{P}_{2} \mathrm{O}_{5}$.

B. Anorganische Phosphate in $100 \mathrm{ccm}$. I. $0,0230 \mathrm{~g}, \mathrm{II} .0,0226 \mathrm{~g}$, Mittel $0,0228 \mathrm{~g} \mathrm{Mg}_{2} \mathrm{P}_{2} \mathrm{O}_{7}=0,01458 \mathrm{~g} \mathrm{P}_{2} \mathrm{O}_{5}$; demnach in $1910 \mathrm{ccm}$. $=0,278 \check{g} \mathrm{~g} \mathrm{P}_{2} \mathrm{O}_{5}$.

C. Organischer Phosphor in $1910 \mathrm{ccm} .=0,0305 \mathrm{~g}_{2} \mathrm{O}_{5}$.

Versuch V.

\begin{tabular}{|c|c|c|c|}
\hline Datum & Harnmenge & $\begin{array}{c}\mathrm{N} \\
\text { in } 10 \mathrm{ccm} .\end{array}$ & $\begin{array}{c}\mathrm{N} \\
\text { pro die }\end{array}$ \\
\hline 7. VI. 1899 & $220 \mathrm{ccm}$ & $29,4 \quad \mathrm{mg}$ & $0,6468 \mathrm{~g}$ \\
\hline 8. VI. 1899 & $220>$ & 31,85 & 0,7007 . \\
\hline 9. VI. 1899 & 225 & 30,8 & 0,693 , \\
\hline 10. VI. 1899 & 240 & 30,1 & 0,7227 . \\
\hline \multirow[t]{2}{*}{ 11. VI. 1899} & 240 & 29,4 & 0,7056 \ \\
\hline & $1145 \mathrm{ccm}$ & & $3,4685 \mathrm{~g}$ \\
\hline
\end{tabular}

Mischharn.

A. Gesammtphosphor in $100 \mathrm{ccm}$. I. $0,0717 \mathrm{~g}$, II. $0,0721 \mathrm{~g}$, Mittel $0,0719 \mathrm{~g} \mathrm{Mg}_{2} \mathrm{P}_{8} \mathrm{O}_{7}=0,04599 \mathrm{~g} \mathrm{P}_{2} \mathrm{O}_{5}$; demnach in $1145 \mathrm{ccm}$. $=$ 0,5266 g $\mathrm{P}_{2} \mathrm{O}_{5}$.

B. Anorganische Phosphate in $100 \mathrm{ccm}$. I. $0,0708 \mathrm{~g}, \mathrm{II} .0,0698 \mathrm{~g}$, Mittel $0,0703 \mathrm{~g} \mathrm{Mg}_{2} \mathrm{P}_{2} \mathrm{O}_{7}=0,04496 \mathrm{~g} \mathrm{P}_{2} \mathrm{O}_{5} ;$ demnach in $1145 \mathrm{ccm}$. $=$ $0, \tilde{5} 148 \& \mathrm{P}_{2} \mathrm{O}_{5}$.

C. Organischer Phosphor in $1145 \mathrm{ccm}$. $=0,0118 \mathrm{~g} \mathrm{P}_{8} \mathrm{O}_{5}$.

Versuch VI.

\begin{tabular}{l|c}
\hline Datum & Harnmenge \\
\hline 20. VI. 1899 & $380 \mathrm{ccm}$. \\
21. VI. 1899 & 320, \\
22. VI. 1899 & 300, \\
23. VI. 1899 & $345>$ \\
24. VI. 1899 & $435>$ \\
& $1780 \mathrm{ccm}$.
\end{tabular}

Mischharn $10 \mathrm{ccm} .=17,5 \mathrm{mg} \mathrm{N}$, $1780 \mathrm{ccm} .=3,115 \mathrm{~g} \mathrm{~N}$.

A. Gesammtphosphor in $100 \mathrm{ccm}$. I. $0,0388 \mathrm{~g}$, II. $0,0381 \mathrm{~g}$, Mittel $0,03845 \mathrm{~g}$ $\mathrm{Mg}_{2} \mathrm{P}_{2} \mathrm{O}_{7}=0,02459 \mathrm{~g} \mathrm{P}_{2} \mathrm{O}_{5}$; demnach in $1780 \mathrm{ccm} .=0,4377 \mathrm{~g} \mathrm{P}_{2} \mathrm{O}_{5}$.

B. Anorganische Phosphate in $100 \mathrm{ccm}$. I. $0,0355 \mathrm{~g}$, II. $0,0361 \mathrm{~g}$, Mittel $0,0358 \mathrm{~g} \mathrm{Mg}_{2} \mathrm{P}_{2} \mathrm{O}_{7}=0,0229 \mathrm{~g} \mathrm{P}_{2} \mathrm{O}_{5}$; demnach in $1780 \mathrm{ccm} .=0,40762 \mathrm{~g} \mathrm{P}_{2} \mathrm{O}_{5}$.

C. Organis cher Phosphor in $1740 \mathrm{ccm}$. $=0,0301 \mathrm{~g} \mathrm{P}_{2} \mathrm{O}_{5}$. 
Versuch VII.

\begin{tabular}{|c|c|c|c|c|}
\hline Datum & Harnmenge & $\begin{array}{c}\mathrm{P}_{2} \mathrm{O}_{5} \\
\text { in } 50 \mathrm{ccm} .\end{array}$ & $\begin{array}{c}\mathrm{P}_{2} \mathrm{O}_{5} \\
\text { pro die }\end{array}$ & \\
\hline 28. VI. 1899 & $230 \mathrm{ccm}$. & 87,075 & 0,4005 & $\mathrm{~g}$ \\
\hline 29. VI. 1899 & 245 , & 173,505 & 0,850175 & 2 \\
\hline 30. VI. 1899 & 310 , & 56,76 & 0,3519 & » \\
\hline 1. VII. 1899 & 335 & 24,8325 & 0,1664 & 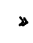 \\
\hline \multirow[t]{2}{*}{ 2. VII. 1899} & 415 & \multirow[t]{2}{*}{14,5} & 0,1189 & $\triangleright$ \\
\hline & $1530 \mathrm{ccm}$. & & 1,8879 & $\mathrm{~g}$ \\
\hline
\end{tabular}

Mischharn $10 . \mathrm{ccm} .=185 \check{\mathrm{mg} \mathrm{N}}, 1530 \mathrm{ccm} .=2,838 \mathrm{~g} \mathrm{~N}$.

A. Gesammtphosphor in $50 \mathrm{ccm}$. I. $0,0991 \mathrm{~g}$, II. $0,0983 \mathrm{~g}$, Mittel $0,0987 \mathrm{~g} \mathrm{Mg}_{2} \mathrm{P}_{2} \mathrm{O}_{7}=0,06313 \mathrm{~g} \mathrm{P}_{2} \mathrm{O}_{5}$; demnach in $1530 \mathrm{ccm}$. = $1,9317 \mathrm{~g} \mathrm{P}_{2} \mathrm{O}_{5}$.

B. Anorganische Phosphate in $50 \mathrm{ccm}$. I. $0,0976 \mathrm{~g}$, II. $0,0976 \mathrm{~g}$ $\mathrm{Mg}_{2} \mathrm{P}_{2} \mathrm{O}_{7}=0,0624 \mathrm{~g} \mathrm{P}_{2} \mathrm{O}_{5}$; demnach in $1530 \mathrm{ccm} .=1,9094 \mathrm{~g} \mathrm{P}_{2} \mathrm{O}_{5}$.

C. Organischer Phosphor in $1530 \mathrm{ccm}$. $=0,0223 \mathrm{~g}_{2} \mathrm{O}_{5}$.

Versuch VIII.

\begin{tabular}{|c|c|}
\hline Datum & Harnmenge \\
\hline 5. VII. 1899 & $550 \mathrm{ccm}$. \\
\hline 6. VII. 1899 & 460 \\
\hline 7. VII. 1899 & 550 \\
\hline 8. VII. 1899 & 580 \\
\hline \multirow[t]{2}{*}{ 9. VII. 1899} & 570 \\
\hline & $2710 \mathrm{ccm}$. \\
\hline
\end{tabular}

Mischharn $10 \mathrm{ccm} .=14,53 \mathrm{mg} \mathrm{N}$, $2710 \mathrm{ccm} .=3,9376 \mathrm{~g} \mathrm{~N}$.

A. Gesammtphosphor in $150 \mathrm{ccm}$. I. $0,0427 \mathrm{~g}$, II. $0,0423 \mathrm{~g}$, Mittel $0,0425 \mathrm{~g}$ $\mathrm{Mg}_{2} \mathrm{P}_{2} \mathrm{O}_{7}=0,0271$ g $\mathrm{P}_{2} \mathrm{O}_{5} ;$ demnach in $2710 \mathrm{~g}=0,4910 \mathrm{~g} \mathrm{P}_{2} \mathrm{O}_{5}$.

B. Anorganische Phosphate in $150 \mathrm{ccm}$. I. $0,0389 \mathrm{~g}$, II. $0,0391 \mathrm{~g}$, Mittel 0,039 g $\mathrm{Mg}_{2} \mathrm{P}_{2} \mathrm{O}_{7}=0,02494$ g $\mathrm{P}_{2} \mathrm{O}_{5}$; demnach in $2710 \mathrm{~g}=0,4506 \mathrm{~g} \mathrm{P}_{2} \mathrm{O}_{5}$.

C. Organischer $\mathrm{Phosphor}$ in $2710 \mathrm{ccm}$. Harn $=0,0404 \mathrm{~g} \mathrm{P}_{2} \mathrm{O}_{5}$. 
Versuch IX.

\begin{tabular}{c|c}
\hline \hline Datum & Harnmenge \\
\hline 12. VII. 1899 & $310 \mathrm{ccm}$. \\
13. VII. 1899 & $340>$ \\
14. VII. 1899 & $270>$ \\
15. VII. 1899 & $450 \gg$ \\
16. VII. 1899 & $430>$ \\
\cline { 2 - 2 } &
\end{tabular}

\begin{tabular}{c|c}
\hline \hline Datum & Harnmenge \\
\hline 12. VII. 1899 & $160 \mathrm{ccm}$. \\
13. VII. 1899 & 200, \\
14. VII. 1899 & 160, \\
15. VII. 1899 & 160, \\
16. VII. 1899 & 225, \\
\cline { 2 - 2 } & $905 \mathrm{ccm}$.
\end{tabular}

\begin{tabular}{c|c}
\hline \hline Datum & Harnmenge \\
\hline 26. VII. 1899 & $370 \mathrm{ccm}$. \\
27. VII. 1899 & 420, \\
28. VII. 1899 & 465, \\
29. VII. 1899 & 315, \\
30. VII. 1899 & 370, \\
\cline { 2 - 2 } & $1940 \mathrm{ccm}$.
\end{tabular}

Mischharn $10 \mathrm{ccm} .=$ I. 100,1 $\mathrm{mg} \mathrm{N}$, II. $99,75 \mathrm{mg} \mathrm{N}$, Mittel $99,975 \mathrm{mg} \mathrm{N}$; also $1800 \mathrm{ccm} .=17,9955 \mathrm{~g} \mathrm{~N}$.

A. Gesammtphosphor in $50 \mathrm{ccm}$. I. $1851 \mathrm{~g}$, II. $0,1845 \mathrm{~g}$, Mittel $0,1848 \mathrm{~g} \mathrm{M}_{2} \mathrm{P}_{2} \mathrm{O}_{7}$ $=0,1182 \mathrm{~g} \mathrm{P}_{2} \mathrm{O}_{5}$; demnach in $1800 \mathrm{ccm}$. $=4,2552 \mathrm{~g} \mathrm{P}_{2} \mathrm{O}_{5}$.

B. Anorganische Phosphate in 亏0 ccm. I. $0,1814 \mathrm{~g}$, II. $0,1805 \mathrm{~g}$, Mittel $0,18095 \mathrm{~g} \mathrm{Mg}_{2} \mathrm{P}_{2} \mathrm{O}_{7}=0,1157 \mathrm{~g} \mathrm{P}_{2} \mathrm{O}_{5}$; demnach in $1800 \mathrm{ccm}$. $=4,1652 \mathrm{~g} \mathrm{P}_{2} \mathrm{O}_{5}$.

C. Organischer Phosphor in $1800 \mathrm{ccm}$. $=0,09 \mathrm{~g} \mathrm{P}_{2} \mathrm{O}_{5}$.

Versuch X.

Mischharn $10 \mathrm{ccm} .=1.47,2 \mathrm{mg}$, II. $48,1 \mathrm{mg}$, Mittel $47,65 \mathrm{mg} \mathrm{N}$; also $905 \mathrm{ccm}$. $=4,3123 \mathrm{~g} \mathrm{~N}$.

A. Gesammtphosphor in $50 \mathrm{ccm}$. I. $0,1828 \mathrm{~g}$, II. $1831 \mathrm{~g}$, Mittel 0,18295 g $\mathrm{Mg}_{2} \mathrm{P}_{2} \mathrm{O}_{7}$ $=0,1170 \mathrm{~g} \mathrm{P}_{2} \mathrm{O}_{5}$; demnach in $905 \mathrm{ccm}$. $=$ 2,1177 g $\mathrm{P}_{2} \mathrm{O}_{5}$.

B. Anorganische Phosphate in $50 \mathrm{ccm}$. I. $0,1822 \mathrm{~g}$, II. $0,1819 \mathrm{~g}$, Mittel $0,18205 \mathrm{~g} \mathrm{Mg}_{2} \mathrm{P}_{2} \mathrm{O}_{7}=0,1164$ g $\mathrm{P}_{2} \mathrm{O}_{5}$; demnach in $905 \mathrm{ccm} .=2,1068 \mathrm{~g} \mathrm{P}_{2} \mathrm{O}_{5}$.

C. Organischer $\mathrm{Phosphor}$ in $905 \mathrm{ccm}$. $=0,0109 \mathrm{~g} \mathrm{P}_{\mathrm{z}} \mathrm{O}_{\mathrm{5}}$.

Versuch XI.

Mischharn $10 \mathrm{ccm}$. $=1575 \mathrm{mg} \mathrm{N}$, $1940 \mathrm{ccm} .=3,0405 \mathrm{~g} \mathrm{~N}$.

A. Gesammtphosphor in $100 \mathrm{ccm}$. I. $0,0467 \mathrm{~g}$, II. 0,0471 g, Mittel 0,0469 g Mg $2 \mathrm{P}_{2} \mathrm{O}_{5}$ $=0,0300 \mathrm{~g} \mathrm{P}_{2} \mathrm{O}_{5}$; demnach in $1940 \mathrm{ccm}$. $=0,582 \mathrm{~g} \mathrm{P}_{2} \mathrm{O}_{5}$.

B. Anorganische Phosphate in $100 \mathrm{ccm}$. I. $0,0448 \mathrm{~g}$, II. $0,0442 \mathrm{~g}$, Mittel 0,0445 g $\mathrm{Mg}_{2} \mathrm{P}_{2} \mathrm{O}_{7}=0,02846$ g $_{2} \mathrm{O}_{5}$; demnach in $1940 \mathrm{ccm} .=0,5521 \mathrm{~g} \mathrm{P}_{2} \mathrm{O}_{5}$.

C. Organischer Phosphor in $1940 \mathrm{ccm}$. $=0,0299 \mathrm{~g} \mathrm{P}_{2} \mathrm{O}_{5}$. 
Versuch XII.

\begin{tabular}{|c|c|}
\hline Datum & Harnmenge. \\
\hline 26. VII. 1899 & $535 \mathrm{ccm}$. \\
\hline 27. VII. 1899 & 700 \\
\hline 28. VII. 1899 & 675 \\
\hline 29. VII. 1899 & 650 \\
\hline \multirow[t]{2}{*}{ 30. VII. 1899} & 650 \\
\hline & $3210 \mathrm{ccm}$. \\
\hline
\end{tabular}

Mischharn. $10 \mathrm{ccm} .=20,65 \mathrm{mg} \mathrm{N}$, $3210 \mathrm{ccm} .=6,62865 \mathrm{~g} \mathrm{~N}$.

A. Gesammtphosphor in $100 \mathrm{ccm}$. I. $0,1000 \mathrm{~g}$, II. $0,1000 \mathrm{~g} \mathrm{Mg}_{2} \mathrm{P}_{2} \mathrm{O}_{7}=0,06396 \mathrm{~g}$ $\mathrm{P}_{2} \mathrm{O}_{5}$; demnach in $3210 \mathrm{ccm} .=2,0531 \mathrm{~g} \mathrm{P}_{2} \mathrm{O}_{5}$.

B. Anorganische Phosphate in $100 \mathrm{ccm}$. I. $0,0988 \mathrm{~g}$, II. $0,0983 \mathrm{~g}$, Mittel $0,09855 \mathrm{~g} \mathrm{Mg}_{2} \mathrm{P}_{2} \mathrm{O}_{7}=0,06303 \mathrm{~g} \mathrm{P}_{2} \mathrm{O}_{5}$; demnach in $3210 \mathrm{ccm} .=2,0233 \mathrm{~g} \mathrm{P}_{2} \mathrm{O}_{5}$.

C. Organis cher Phosphor in $3210 \mathrm{ccm}$. Harn $=0,0298$ g $\mathrm{P}_{2} \mathrm{O}_{5}$.

Versuch XIII.

$\mathrm{M}$ is chharn $10 \mathrm{ccm} .=0,0222 \mathrm{~g} \mathrm{~N}$, $2110 \mathrm{ccm} .=4,6895 \mathrm{~g} \mathrm{~N}$.

A. Gesammtphosphor in $50 \mathrm{ccm}$. I. $0,0670 \mathrm{~g}$, II. $0,0676 \mathrm{~g}$, Mittel $0,0673 \mathrm{~g}$ $\mathrm{Mg}_{2} \mathrm{P}_{2} \mathrm{O}_{7}=0,0430 \mathrm{~g} \quad \mathrm{P}_{2} \mathrm{O}_{5}$; demnach in $2110 \mathrm{ccm} .=1,8146 \mathrm{~g} \mathrm{P} \mathrm{P}_{5}$.

B. Anorganische Phosphate in $50 \mathrm{ccm}$. I. 0,0644 g, II. $0,0640 \mathrm{~g}$, Mittel 0,0642 g $\mathrm{Mg}_{2} \mathrm{P}_{2} \mathrm{O}_{7}=0,0411$ g $\mathrm{P}_{2} \mathrm{O}_{5}$; demnach in $2110 \mathrm{ccm} .=1,7344 \mathrm{~g}_{2} \mathrm{O}_{5}$.

C. Organischer Phosphor in $2110 \mathrm{ccm}$. $=0,0802 \mathrm{~g} \mathrm{P}_{2} \mathrm{O}_{5}$.

Hungerversuch am Kind.

24.-25. Juli 1899.

$620 \mathrm{ccm}$. Harn.

$10 \mathrm{ccm} .=27,825 \mathrm{mg} \mathrm{N}, 620 \mathrm{ccm} .=1,72515 \mathrm{~g} \mathrm{~N}$.

A. Gesammtphosphor in $300 \mathrm{ccm}$. $0,3253 \mathrm{~g} \mathrm{Mg}_{2} \mathrm{P}_{2} \mathrm{O}_{7}=0,2081 \mathrm{~g}$ $\mathrm{P}_{2} \mathrm{O}_{5}$; demnach in $620 \mathrm{ccm}$. $=0,4308 \mathrm{~g} \mathrm{P}_{2} \mathrm{O}_{5}$.

B. Anorganische Phosphate in $300 \mathrm{ccm}$. $0,3192 \mathrm{~g}_{\mathrm{Mg}_{2}} \mathrm{P}_{2} \mathrm{O}_{7}$ $=0,2042 \mathrm{~g} \mathrm{P}_{2} \mathrm{O}_{5}$; demnach in $620 \mathrm{ccm}$. $=0,4247 \mathrm{~g} \mathrm{P}_{2} \mathrm{O}_{5}$.

C. Organischer Phosphor in $620 \mathrm{ccm} .=0,0061 \mathrm{~g} \mathrm{P}_{2} \mathrm{O}_{5}$.

Darreichung von Protogen.

25.-28. Juli 1899.

$1 \mathrm{~g}$ Protogen $=$ I. $0,0125 \mathrm{~g}$, II. $0,0128 \mathrm{~g}$, Mittel 0,01265 g $\mathrm{Mg}_{2} \mathrm{P}_{2} \mathrm{O}_{7}$ $=0,00809 \mathrm{~g} \mathrm{P}_{2} \mathrm{O}_{5}$. 


\begin{tabular}{|c|c|}
\hline Datum & Harnmenge \\
\hline 25.-26. VII. 1899 & $1070 \mathrm{ccm}$. \\
\hline 26.-27. VII. 1899 & 1010 \\
\hline 27...-28. VII. 1899 & 1260 \\
\hline & $3340 \mathrm{ccm}$. \\
\hline
\end{tabular}

Mischharn.

$10 \mathrm{ccm} .=15,05 \mathrm{mg} \cdot \mathrm{N}$,

$3340 \gg=\check{5}, 0267 \mathrm{~g} \mathrm{~N}$.

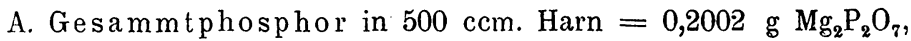
$200 \mathrm{ccm}$. Harn $=0,0800 \mathrm{~g} \mathrm{Mg}_{2} \mathrm{P}_{2} \mathrm{O}_{7}, 200 \mathrm{ccm}$. Harn $=0,0810 \mathrm{~g} \mathrm{Mg}_{2} \mathrm{P}_{2} \mathrm{O}_{7}$; also enthalten $100 \mathrm{ccm}$. Harn I. $0,04004 \mathrm{~g}$, II. $0,0400 \mathrm{~g}$, III. $0,0405 \mathrm{~g}$, Mittel $0,04018 \mathrm{~g} \mathrm{Mg}_{2} \mathrm{P}_{2} \mathrm{O}_{7}, 3340 \mathrm{ccm} .=1,3420 \mathrm{~g} \mathrm{Mg}_{2} \mathrm{P}_{2} \mathrm{O}_{7}=0,8583 \mathrm{~g} \mathrm{P}_{2} \mathrm{O}_{5}$.

B. Anorganische Phosphate in $300 \mathrm{ccm} .=0,1168 \mathrm{~g}_{\mathrm{Mg}_{2}} \mathrm{P}_{2} \mathrm{O}_{7}$, $500 \mathrm{ccm} .=0,1970 \mathrm{~g} \mathrm{Mg}_{2} \mathrm{P}_{2} \mathrm{O}_{7}, 200 \mathrm{ccm} .=0,0782 \mathrm{~g} \mathrm{Mg}_{2} \mathrm{P}_{2} \mathrm{O}_{7}, 200 \mathrm{ccm}$. $=0,0792 \mathrm{~g} \mathrm{Mg}_{2} \mathrm{P}_{2} \mathrm{O}_{7} ;$ also enthalten $100 \mathrm{ccm}$. I. $0,0389 \mathrm{~g}$, II. $0,0394 \mathrm{~g}$, III. $0,0391 \mathrm{~g}$; IV. $0,0386 \mathrm{~g}$, Mittel $0,0390 \mathrm{~g} \mathrm{Mg} \mathrm{P}_{2} \mathrm{O}_{7} .3340 \mathrm{ccm} .=1,3026 \mathrm{~g}$ $\mathrm{Mg}_{2} \mathrm{P}_{2} \mathrm{O}_{7}=0,8331 \mathrm{~g} \mathrm{P}_{2} \mathrm{O}_{5}$.

C. Organischer Phosphor in $3340 \mathrm{ccm}$. $=0,0252 \mathrm{~g} \mathrm{P}_{2} \mathrm{O}_{5}$.

28.-29. Juli 1899.

Harnmenge $1520 \mathrm{ccm} .10 \mathrm{ccm} .=9,275 \mathrm{mg} \mathrm{N}, 1520 \mathrm{ccm} .1,4098 \mathrm{~g} \mathrm{~N}$.

A. Gesammtphosphor in $300 \mathrm{ccm}$. I. $0,0657 \mathrm{~g}$ II. $0,0663 \mathrm{~g}$, Mittel $0,0660 \mathrm{~g} \mathrm{Mg}_{2} \mathrm{P}_{2} \mathrm{O}_{7}=0,04221 \mathrm{~g} \mathrm{P}_{2} \mathrm{O}_{5}$; demnach in $1520 \mathrm{ccm}$. $=0,2140 \mathrm{~g} \mathrm{P}_{2} \mathrm{O}_{5}$.

B. Anorganische Phosphate in $300 \mathrm{ccm}$. I. $0,0635 \mathrm{~g}$, II. $0,0635 \mathrm{~g}$ $\mathrm{Mg}_{2} \mathrm{P}_{2} \mathrm{O}_{7}=0,04061 \mathrm{~g}_{2} \mathrm{O}_{5}$; demnach in $1520 \mathrm{ccm} .=0,2059 \mathrm{~g} \mathrm{P}_{\mathbf{2}} \mathrm{O}_{5}$.

C. Organischer Phosphor in $1520 \mathrm{ccm}$. Harn $=0,0081 \mathrm{~g} \mathrm{P}_{2} \mathrm{O}_{5}$.

\section{August 1899.}

Harnmenge von 12 Stunden $700 \mathrm{ccm}$.

A. Gesammtphosphor in $150 \mathrm{ccm}$. I. $0,0621 \mathrm{~g}$, II. $0,0618 \mathrm{~g}$, Mittel $0,06195 \mathrm{~g} \mathrm{Mg}_{8} \mathrm{P}_{2} \mathrm{O}_{7}=0,03962 \mathrm{~g} \mathrm{P}_{2} \mathrm{O}_{5}$; demnach in $700 \mathrm{ccm}$. $=0,1806 \% \mathrm{P}_{2} \mathrm{O}_{5}$.

B. Anorganische Phosphate in $150 \mathrm{ccm}$. I. 0,0603 g, II. $0,0610 \mathrm{~g}$, Mittel $0,06065 \mathrm{~g} \mathrm{Mg}_{2} \mathrm{P}_{2} \mathrm{O}_{7}=0,03879 \mathrm{~g} \mathrm{P}_{2} \mathrm{O}_{5}$; demnach in 700 ccill. $=0,1806 \mathrm{~g} \mathrm{P}_{2} \mathrm{O}_{5}$.

C. Organischer Phosphor in $700 \mathrm{ccm}$. $=0,0043 \mathrm{~g}_{2} \mathrm{O}_{5}$.

12.-13. August 1899.

Harnmenge $820 \mathrm{ccm} .10 \mathrm{ccm} .=16,8 \mathrm{mg} \mathrm{N}, 820 \mathrm{ccm} .=1,3776 \mathrm{~g} \mathrm{~N}$.

A. Gesam mtphosphor in $150 \mathrm{ccm}$. I. $0,0931 \mathrm{~g}$, II. $0,0941 \mathrm{~g}$, Mittel $0,0936 \mathrm{~g} \mathrm{Mg}_{2} \mathrm{P}_{2} \mathrm{O}_{7}=0,05987 \mathrm{~g} \mathrm{P}_{2} \mathrm{O}_{5} ;$ demnach in $820 \mathrm{ccm}$. $=0,3293 \mathrm{~g} \mathrm{P}_{2} \mathrm{O}_{5}$.

B. Anorganische Phosphate in $150 \mathrm{ccm}$. I. $0,0920 \mathrm{~g}$, II. $0,0915 \mathrm{~g}$, Mittel $0,09175 \mathrm{~g} \mathrm{Mg}_{2} \mathrm{P}_{2} \mathrm{O}_{2}=0,0585 \mathrm{~g} \mathrm{P}_{2} \mathrm{O}_{5}$; demnach in $820 \mathrm{ccm} .=0,3219 \mathrm{~g} \mathrm{P}_{2} \mathrm{O}_{5}$.

C. Organischer Phosphor in $820 \mathrm{ccm}$. Harn $=0,0074 \mathrm{~g} \mathrm{P}_{8} \mathrm{O}_{5}$. 
Selbstversuch.

14.-15. August 1899.

Harnmenge $1020 \mathrm{ccm} .10 \mathrm{ccm} .=80,5 \mathrm{mg} \mathrm{N}, 1020 \mathrm{ccm} .=8,211 \mathrm{~g} \mathrm{~N}$.

A. Gesammtphosphor in $50 \mathrm{ccm}$. I. $0,2231 \mathrm{~g}$, II. $0,2222 \mathrm{~g}$, Mittel $0,22265 \mathrm{~g}_{\mathrm{Mg}_{2}} \mathrm{P}_{2} \mathrm{O}_{7}=0,1424 \mathrm{~g} \mathrm{P}_{2} \mathrm{O}_{5}$; demnach in $1020 \mathrm{ccm}$. $=1,858 \mathrm{~g} \mathrm{P}_{2} \mathrm{O}_{5}$.

B. Anorganische Phosphate in $50 \mathrm{ccm}$. I. $0,2205 \mathrm{~g}$, II. $0,2207 \mathrm{~g}$, Mittel $0,2206 \mathrm{~g} \mathrm{Mg}_{2} \mathrm{P}_{2} \mathrm{O}_{7}=0,1411 \mathrm{~g} \mathrm{P}_{2} \mathrm{O}_{5}$; demnach in $1020 \mathrm{ccm} .=1,8410 \mathrm{~g} \mathrm{P}_{2} \mathrm{O}_{5}$.

C. Organischer Phosphor in $1020 \mathrm{ccm}$. $=0,0170 \mathrm{~g}_{2} \mathrm{O}_{5}$. 15.-16. August 1899.

Harnmenge $460 \mathrm{ccm} .10 \mathrm{ccm} .=146,65 \mathrm{mg} \mathrm{N}, 460 \mathrm{ccm}$. $=6,7459 \mathrm{~g} \mathrm{~N}$.

A. Gesammtphosphor in $50 \mathrm{ccm}$. I. $0,3236 \mathrm{~g}$, II. $0,3228 \mathrm{~g}$, Mittel 0,3232 g $\mathrm{Mg}_{2} \mathrm{P}_{2} \mathrm{O}_{7}=0,2067 \mathrm{~g} \mathrm{P}_{2} \mathrm{O}_{5} ;$ demnach in $460 \mathrm{ccm}$. $=1,9016 \mathrm{~g} \mathrm{P}_{2} \mathrm{O}_{5}$.

B. Anorganische Phosphate in $50 \mathrm{ccm}$. I. $0,3182 \mathrm{~g}$, II. $0,3180 \mathrm{~g}$, Mittel $0,3181 \mathrm{~g} \mathrm{Mg}_{2} \mathrm{P}_{2} \mathrm{O}_{7}=0,2035 \mathrm{~g} \mathrm{P}_{2} \mathrm{O}_{5}$; demnach in $460 \mathrm{ccm} .=1,8722 \mathrm{~g} \mathrm{P}_{2} \mathrm{O}_{5}$.

C. Organischer Phosphor in $460 \mathrm{ccm} .=0,0294 \mathrm{~g} \mathrm{P}_{2} \mathrm{O}_{5}$. 16.-17. August 1899.

Harnmenge $1220 \mathrm{ccm} .10 \mathrm{ccm} .=64,85 \mathrm{mg} \mathrm{N}, 1220 \mathrm{ccm} .=7,9117 \mathrm{~g} \mathrm{~N}$.

A. Gesammtphosphor in $50 \mathrm{ccm}$. I. $0,1566 \mathrm{~g}$, II. $0,1560 \mathrm{~g}$, Mittel $0,1563 \mathrm{~g} \mathrm{Mg}_{2} \mathrm{P}_{2} \mathrm{O}_{7}=0,09997 \mathrm{~g} \mathrm{P}_{2} \mathrm{O}_{5}$; demnach in $1220 \mathrm{ccm}$. $=2,4393 \mathrm{~g} \mathrm{P}_{2} \mathrm{O}_{5}$.

B. Anorganische Phosphate in $50 \mathrm{ccm}$. I. $0,1544 \mathrm{~g}$, II. $0,1538 \mathrm{~g}$, Mittel $0,1541 \mathrm{~g} \mathrm{Mg}_{2} \mathrm{P}_{2} \mathrm{O}_{7}=0,09856 \mathrm{~g} \mathrm{P}_{2} \mathrm{O}_{5}$; demnach in $1220 \mathrm{ccm} .=2,4049 \mathrm{~g} \mathrm{P}_{2} \mathrm{O}_{6}$.

C. Organischer Phosphor in $1220 \mathrm{ccm}$. $=0,0344 \mathrm{~g} \mathrm{P}_{8} \mathrm{O}_{5}$.

$$
\text { 17.-18. August } 1899 .
$$

Harnmenge $1540 \mathrm{ccm} .10 \mathrm{ccm} .=74,55 \mathrm{mg} \mathrm{N}, 1540 \mathrm{ccm} .=11,4807 \mathrm{~g} \mathrm{~N}$.

A. Gesammtphosphor in $50 \mathrm{ccm}$. I. $0,1286 \mathrm{~g}$, II. $0,1279 \mathrm{~g}$, Mittel $0,12825 \mathrm{~g} \mathrm{Mg}_{2} \mathrm{P}_{2} \mathrm{O}_{7}=0,08203 \mathrm{~g} \mathrm{P}_{2} \mathrm{O}_{5} ;$ demnach in $1540 \mathrm{ccm}$. $=2,5265 \mathrm{~g} \mathrm{P}_{2} \mathrm{O}_{5}$.

B. Anorganische Phosphate in $50 \mathrm{ccm}$. I. $0,1253 \mathrm{~g}$, II. $0,1257 \mathrm{~g}$, Mittel $0,1255 \mathrm{Mg}_{2} \mathrm{P}_{2} \mathrm{O}_{7}=0,08017 \mathrm{~g} \mathrm{P}_{2} \mathrm{O}_{5}$; demnach in $1540 \mathrm{ccm} .=2,4692 \mathrm{~g} \mathrm{P}_{2} \mathrm{O}_{5}$.

C. Organischer Phosphor in $1540 \mathrm{ccm} .=0,0573 \mathrm{~g} \mathrm{P}_{2} \mathrm{O}_{5}$. 